\title{
Determination of the Absolute Configuration of
}

\author{
three as-hydrindacene compounds by Vibrational
}

\section{Circular Dichroism}

Kuppens T., Vandyck K., Van der Eycken J., Herrebout W., van der Veken B.J., Bultinck P.

General experimental methods

${ }^{13} \mathrm{C}$ NMR (APT) spectrum of 5

${ }^{1} \mathrm{H}$ NMR spectrum of 5 . S6

${ }^{1} \mathrm{H}$ NMR spectrum of 3 S7

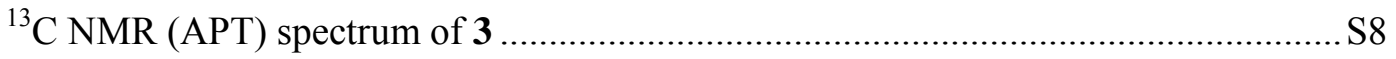

${ }^{1} \mathrm{H}-\mathrm{NMR}$ of epoxidation mixture with $\mathrm{Mn}-(R, R)$-Salen as catalyst ............................. 9

HPLC on Chiralcel OD-H column. detector: $214 \mathrm{~nm}$

$n$-hexane/EtOH 99/1, epoxidation mixture with $\mathrm{Mn}-(R, R)-$ Salen as catalyst...... S9

$n$-hexane/EtOH 99/1, epoxidation mixture with $\mathrm{Mn}-(S, S)$-Salen as catalyst..... S10

n-hexane/EtOH 99/1, mixture of a) epoxidation mixture with Mn-(S,S)-

Salen as catalyst and bisepoxide 3 from epoxidation with Mn-(R,R)-Salen as catalyst.

$n$-hexane/EtOH 99/1, epoxidation mixture with Mn- $(R, R)$-Salen as catalyst without the addition of NMO 
$n$-hexane/EtOH 95/5, reaction epoxidation mixture with Mn- $(R, R)$-Salen as catalyst, without the addition of NMO

$n$-hexane/EtOH 95/5, bisepoxide 3 from epoxidation with Mn- $(R, R)$-Salen as catalyst after crystallisation from toluene

IR absorption and VCD spectrum for (+)-1, (+)-2 and (-)-3, including Lorentzian fit

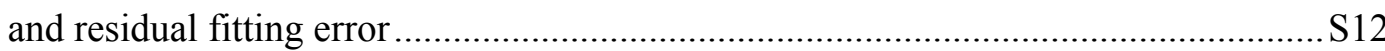

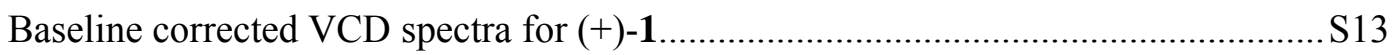

Neighbourhood similarity $\mathrm{S}_{\mathrm{fg}}$ for theoretical versus experimental IR and VCD

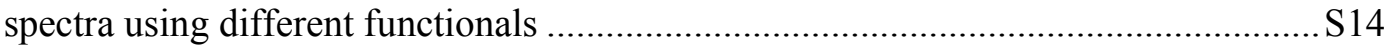

Assignment of the experimental peaks of (+)-1 (B3LYP/6-31G*) ............................ 16

Assignment of the experimental peaks of (+)-1 (B3LYP/cc-pVTZ) ............................ 18

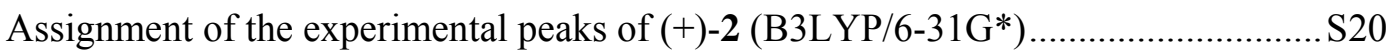

Assignment of the experimental peaks of (+)-2 (B3LYP/cc-pVTZ) .......................... S21

Assignment of the experimental peaks of (-)-3 (B3LYP/6-31G*) .......................... S22

Assignment of the experimental peaks of (-)-3 (B3LYP/cc-pVTZ) .........................S23

Cartesian coordinates for the optimized structures for compound 1........................ S24

Total B3LYP/6-31G* energy for the optimized structures for compound 1 .............. S31

Cartesian coordinates for the B3LYP/6-31G* optimized structures for compound 2. S32 Cartesian coordinates for the B3LYP/6-31G* optimized structure for conformation eG' interacting with $\mathrm{CHCl}_{3}$

Total B3LYP/6-31G* energy for the optimized structures for compound 2

Cartesian coordinates for the B3LYP/6-31G* optimized structure and energy

for compound 3

Experimental data for $(1 S, 8 S)$-1,2,3,6,7,8-Hexahydro-as-indacene-1,8-diol 1 and

(8S)-8-hydroxy-3,6,7,8-tetrahydro-2H-as-indacen-1-one 2 S36

${ }^{13} \mathrm{C}$ NMR (APT) spectrum of $\mathbf{1}$ S38

${ }^{13} \mathrm{C}$ NMR (APT) spectrum of 2 


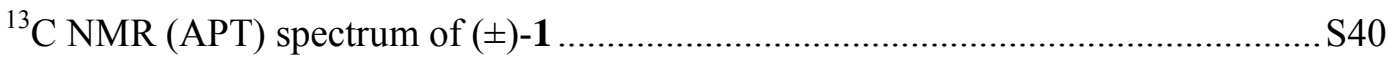




\section{General experimental methods}

All reactions were carried out under an argon atmosphere with magnetic stirring. All solvents were purified or dried according to standard procedures. m-CPBA was purified from a commercial $70-75 \%$ mixture using a known procedure. ${ }^{36}$ Column chromatographic separations were performed with silica gel. ${ }^{1} \mathrm{H}$ NMR spectra were recorded at $300 \mathrm{MHz}$ or at $500 \mathrm{MHz} .{ }^{13} \mathrm{C}$ NMR spectra were recorded at $75 \mathrm{MHz}$. HRMS were recorded with a Q-Tof-2, equipped with a standard electrospray ionization (ESI) interface. 


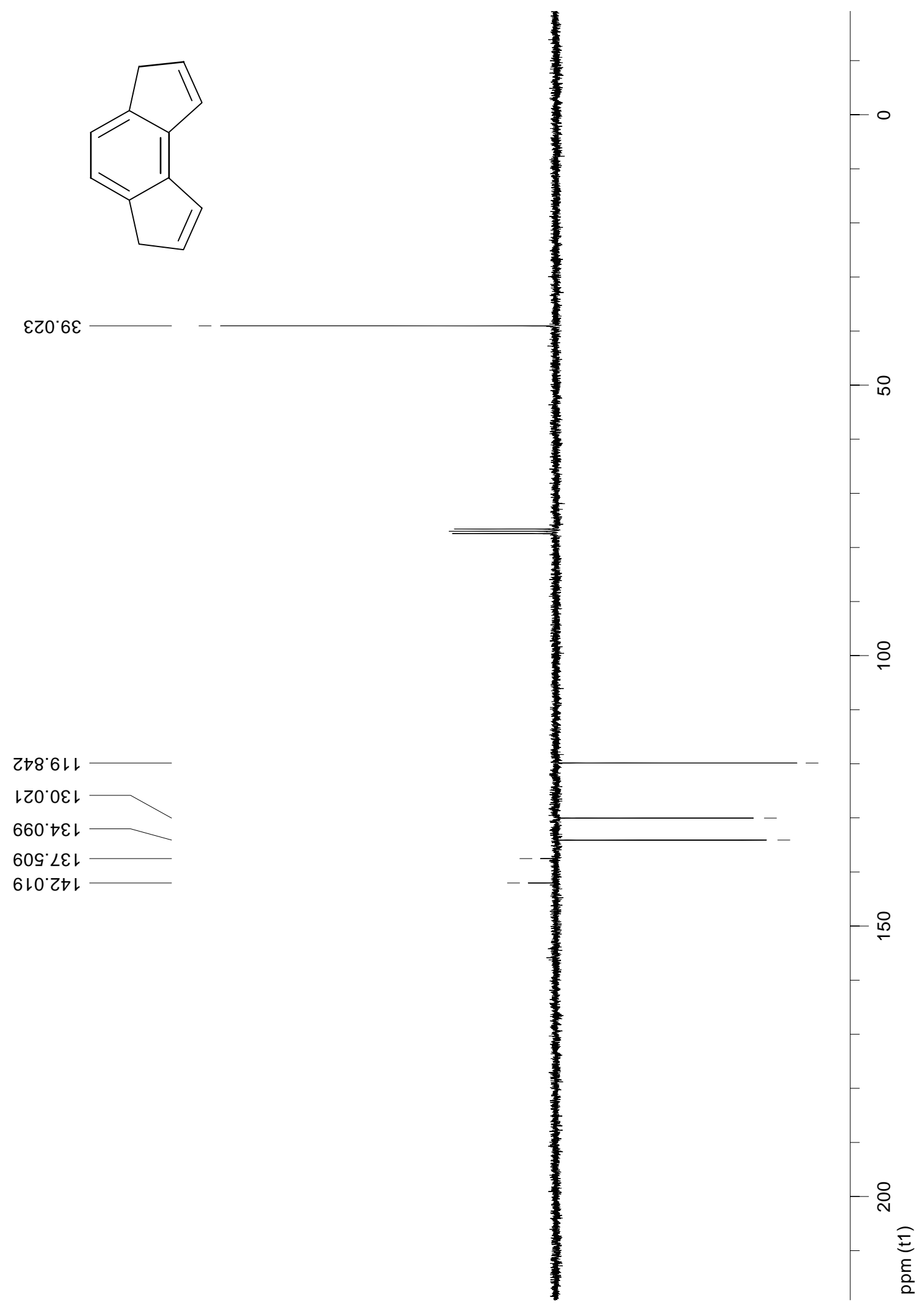




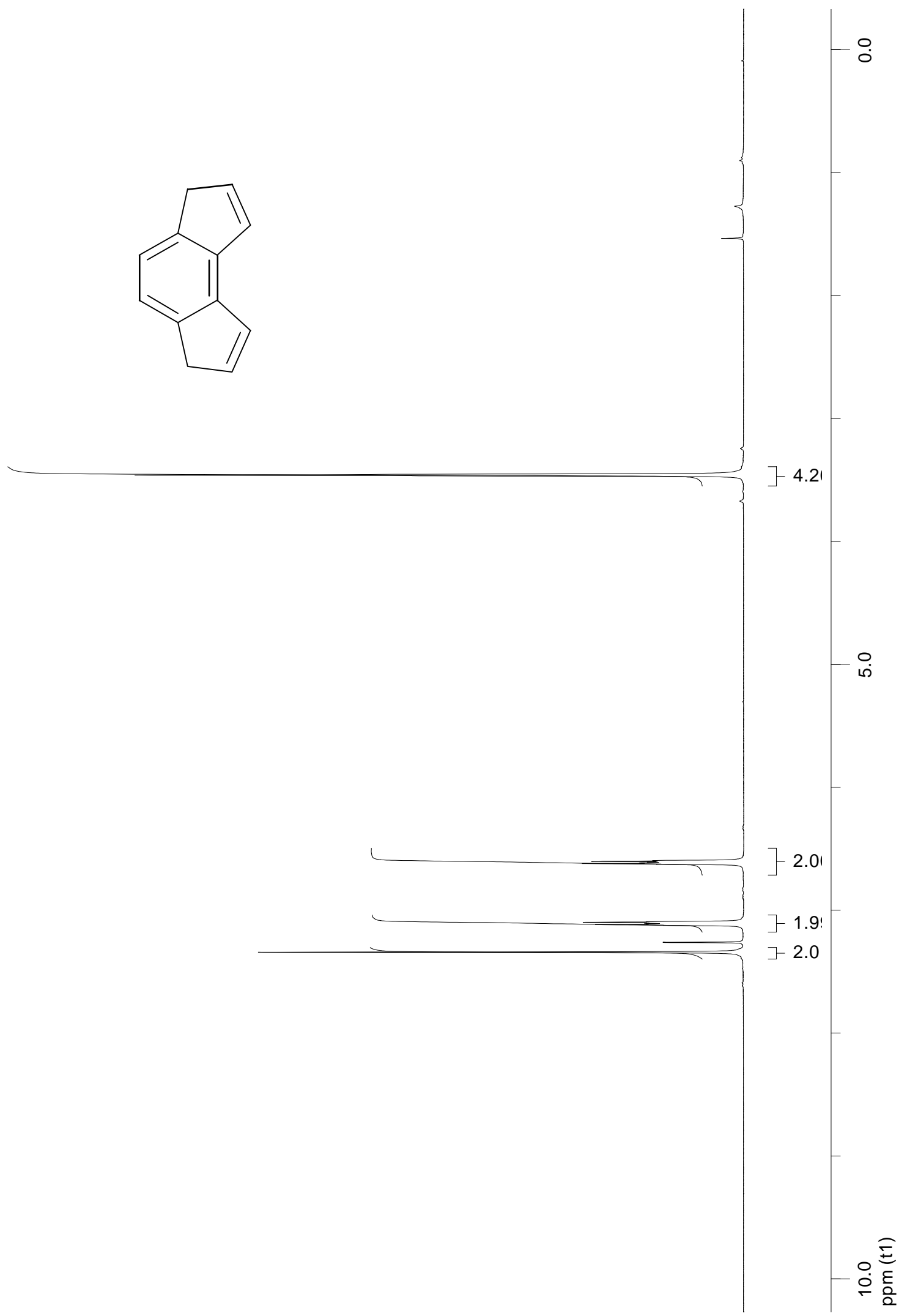

S6 


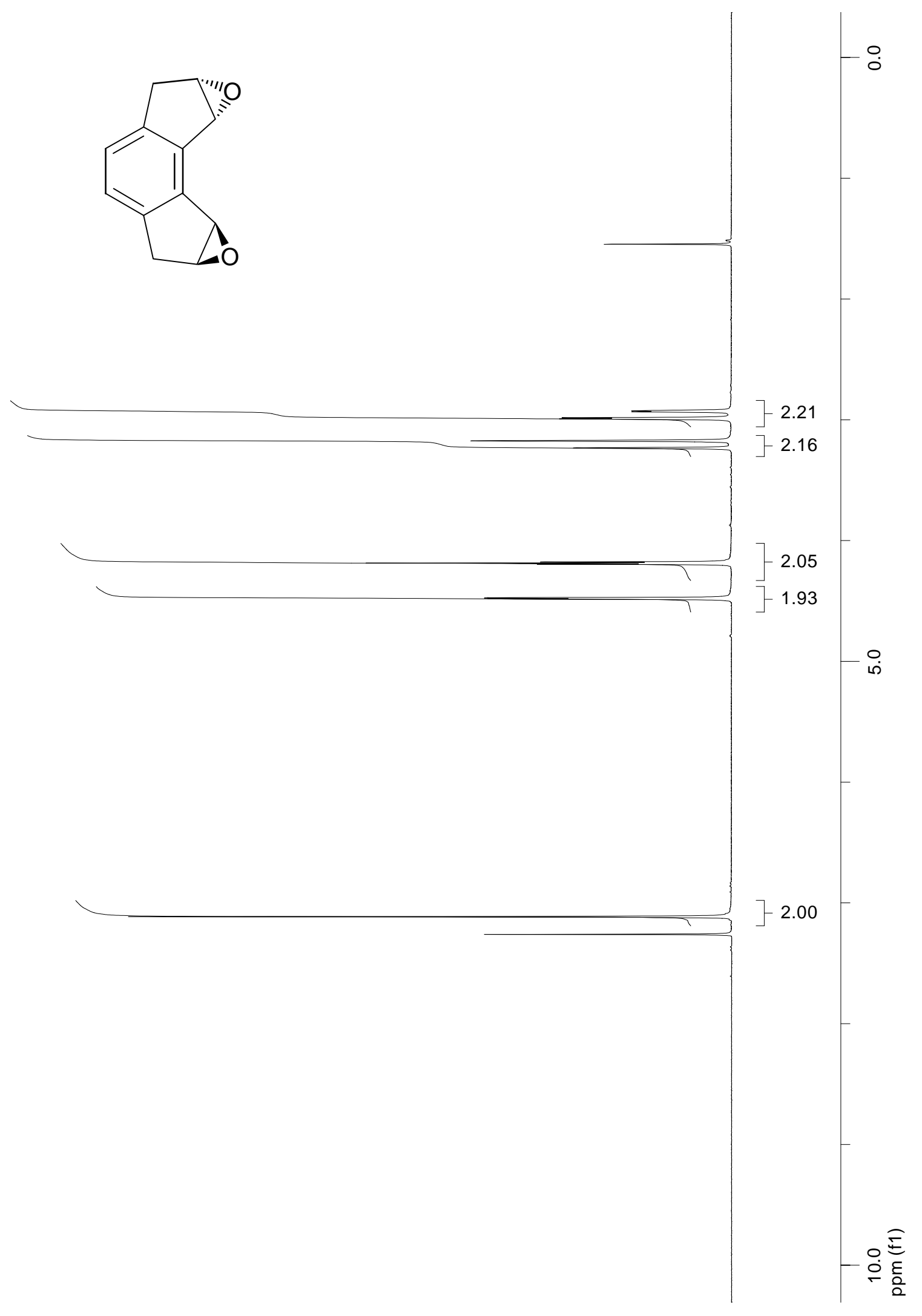




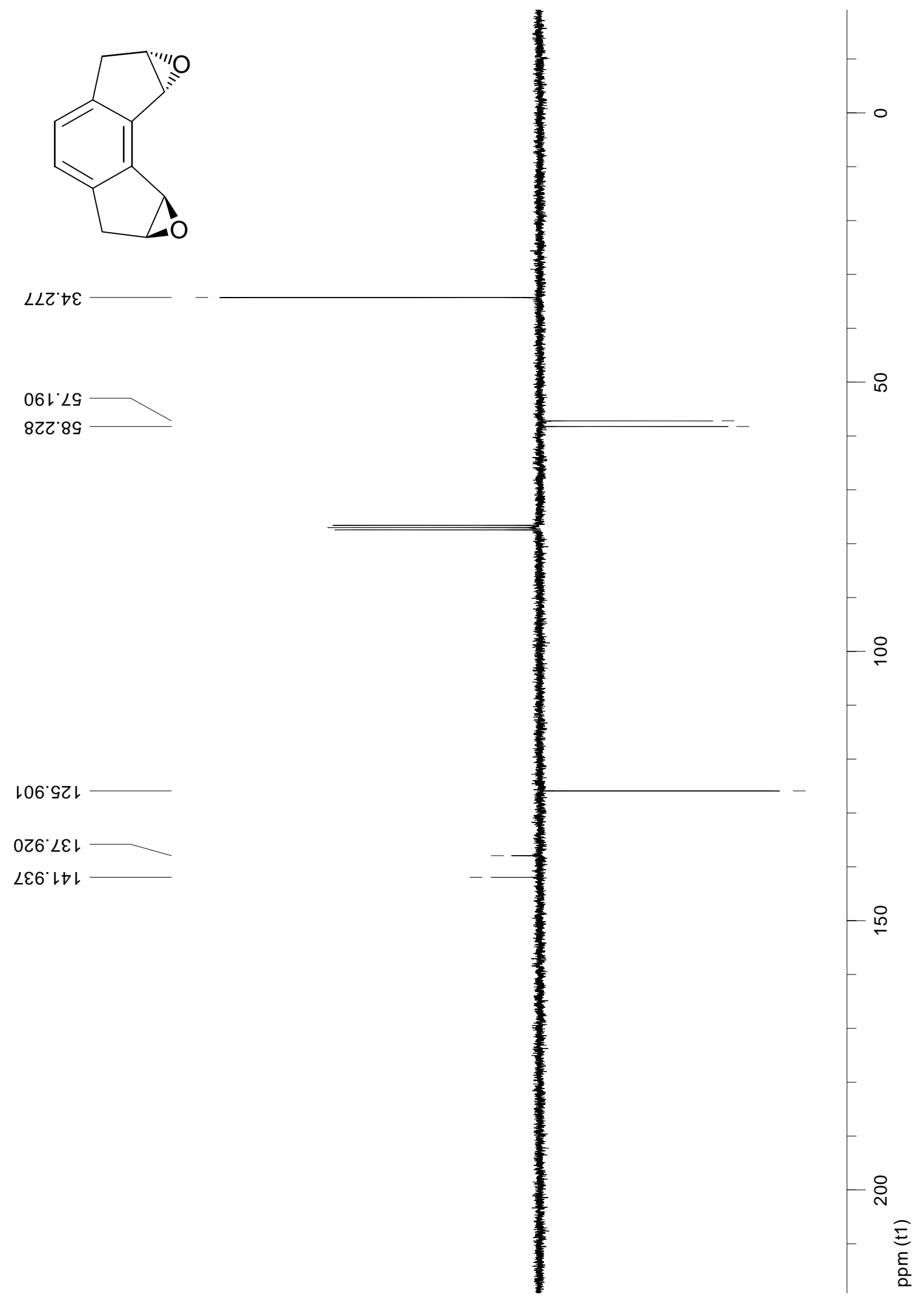




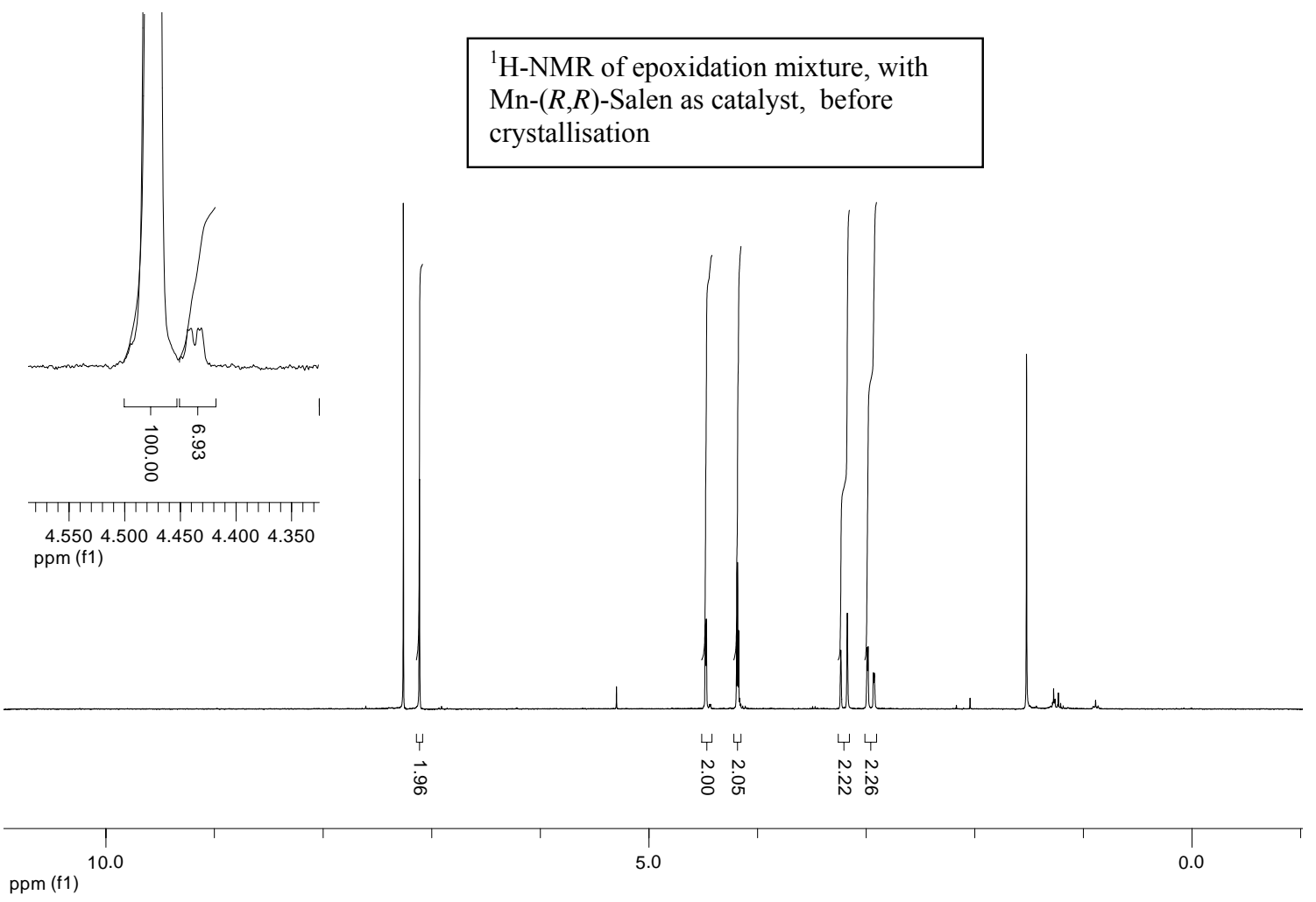

HPLC on Chiralcel OD-H column. detector: $214 \mathrm{~nm}$

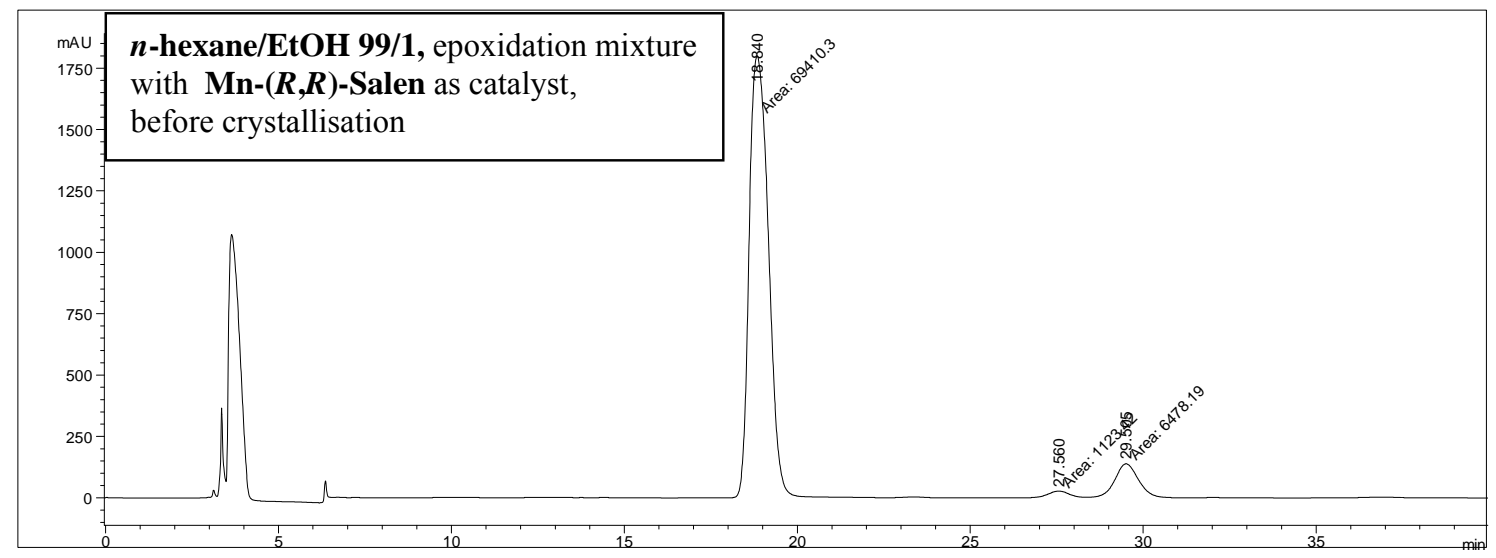



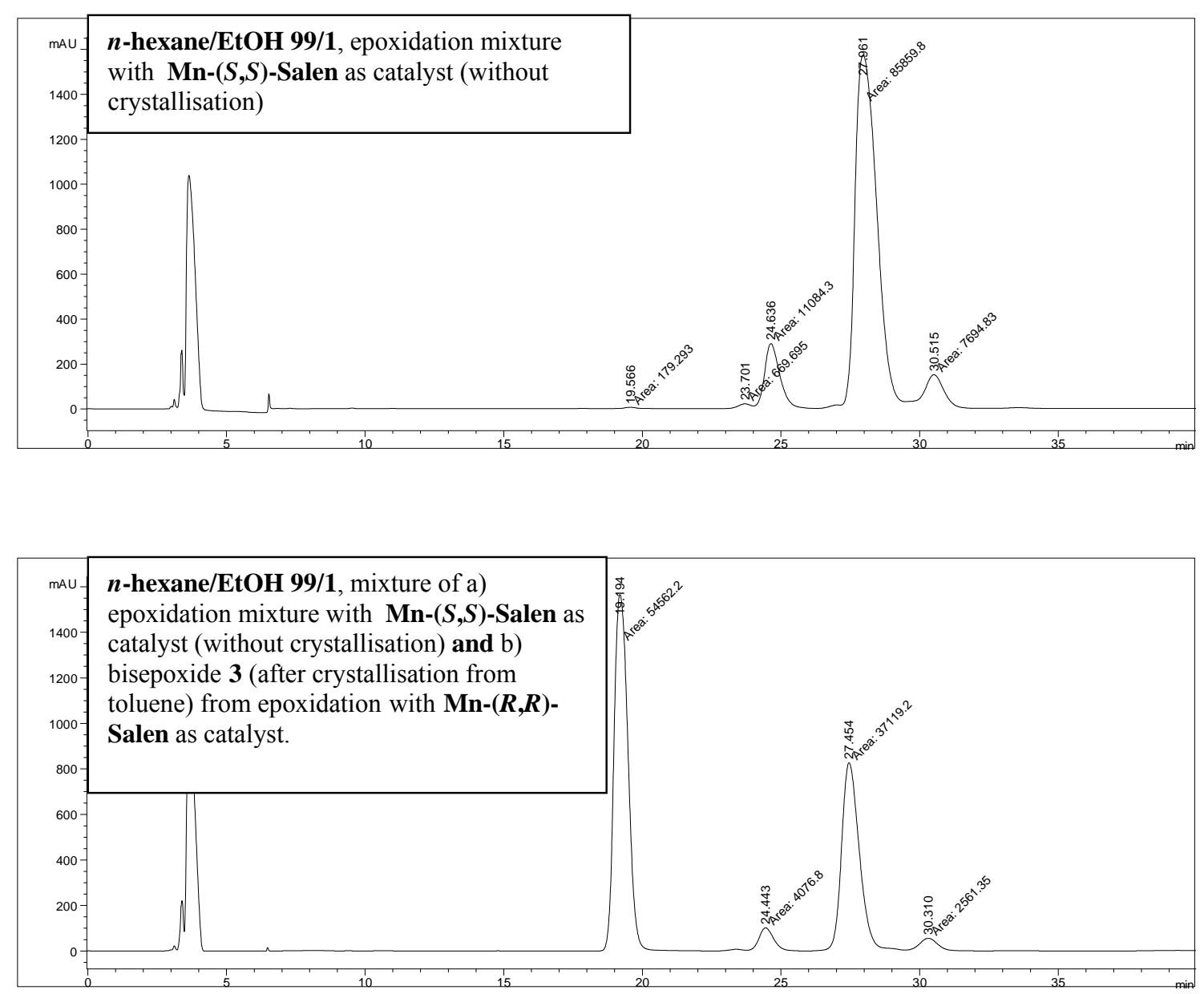

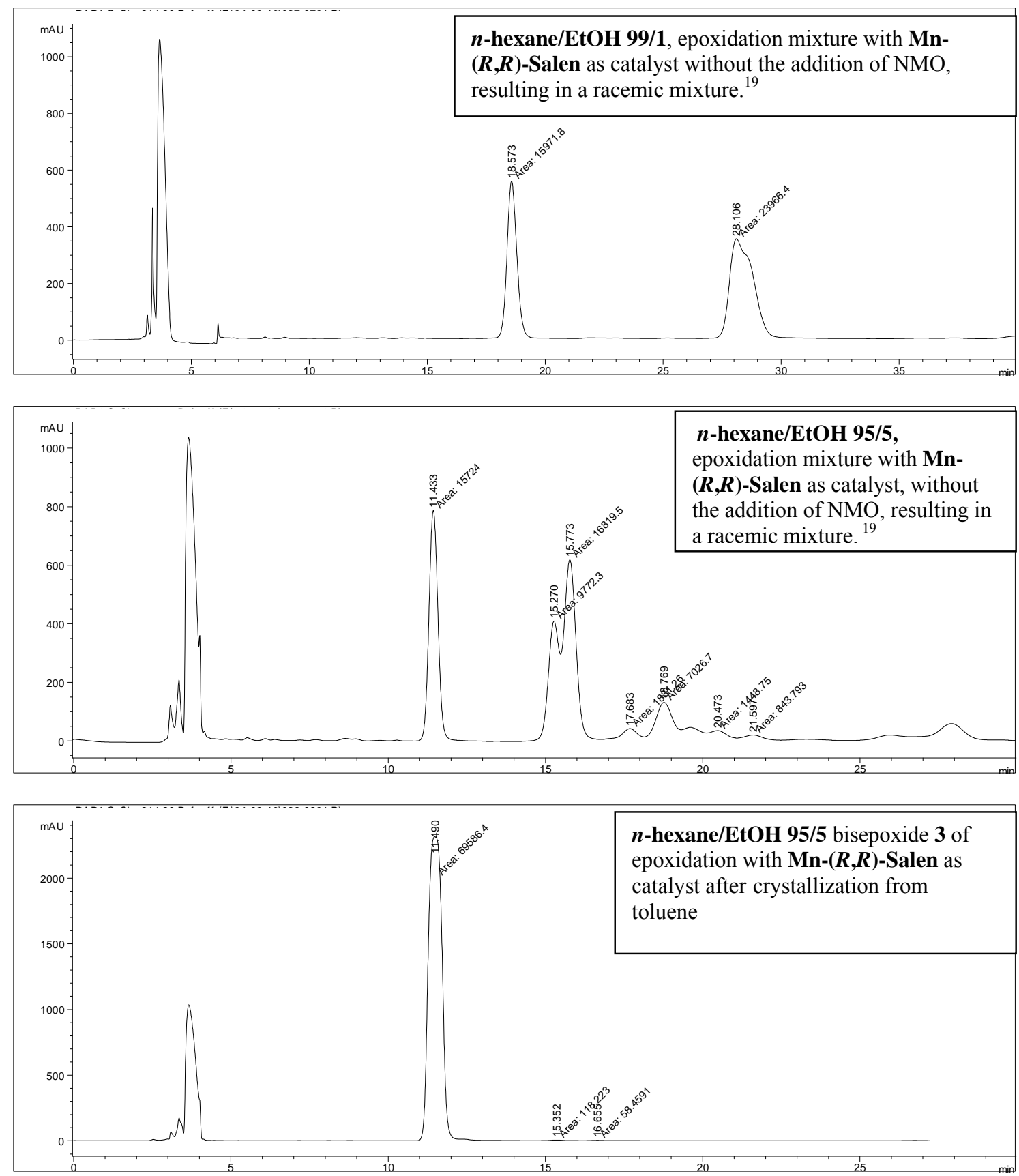
Unpolarized IR absorption and VCD spectrum for (+)-1, (+)-2 and (-)-3, including Lorentzian fit and residual fitting error (absorptions and differential absorptions in absorbance units).
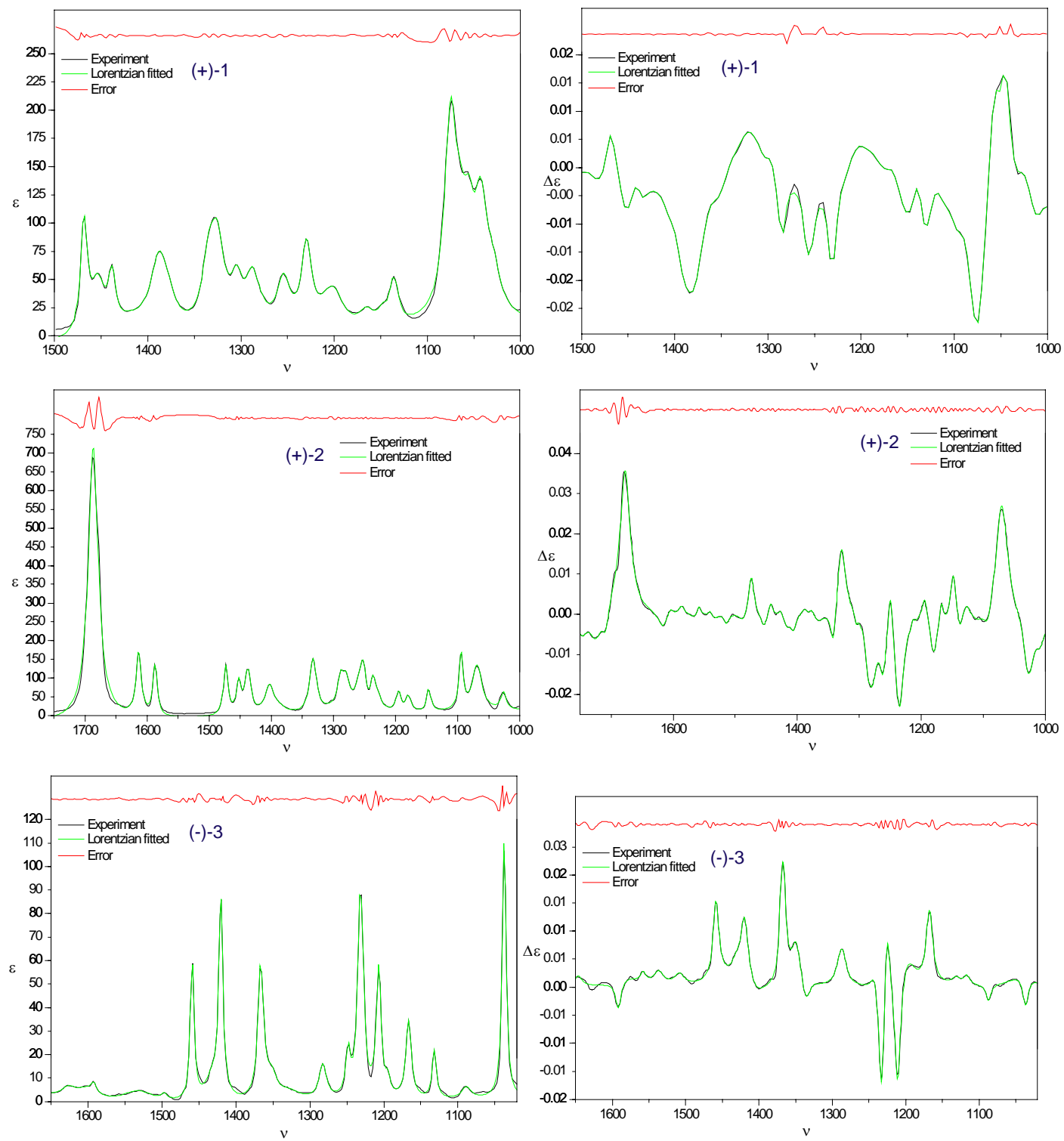
Baseline corrected VCD spectra for $(+)-1$. Black spectrum is solvent corrected, red spectrum is racemic corrected. Differential intensities are in absorbance units, frequencies in $\mathrm{cm}^{-1}$.

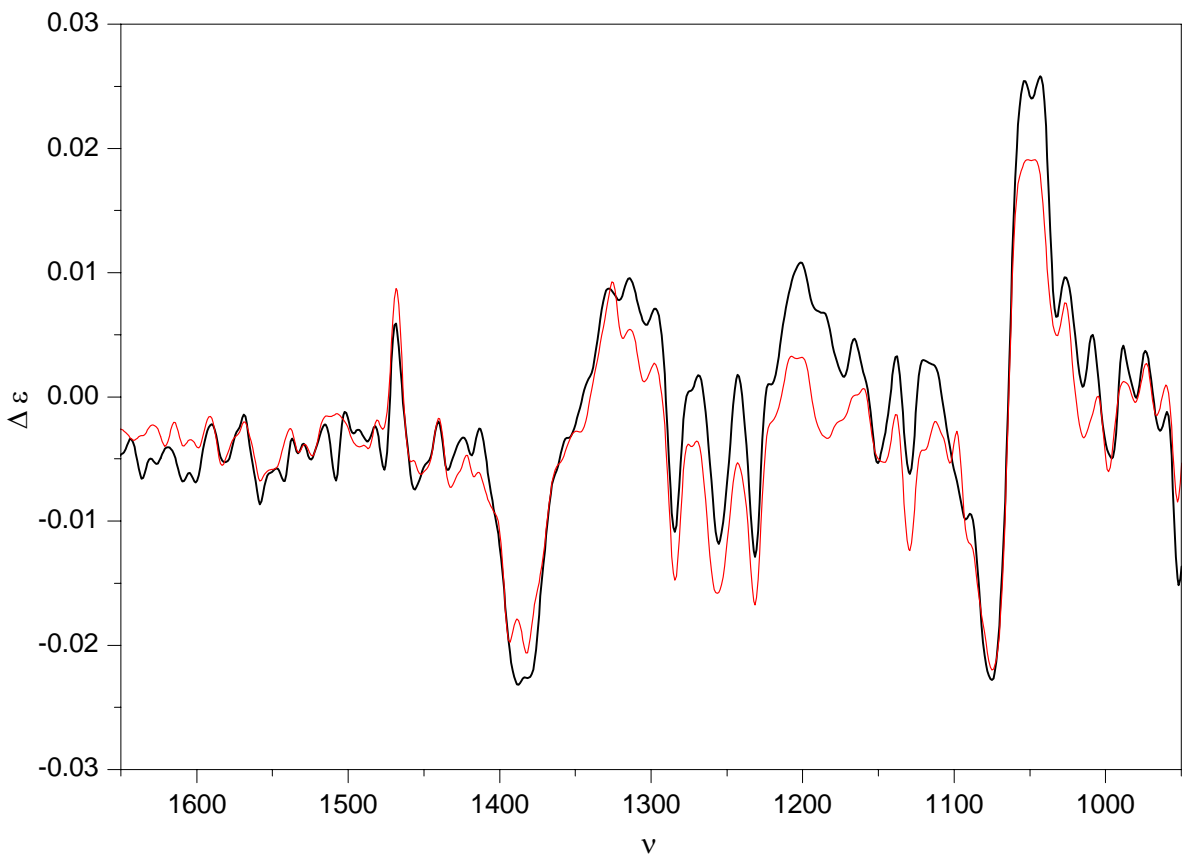


Neighbourhood similarity $\mathrm{S}_{\mathrm{fg}}$ (equation 2) and maximizing scaling factor for theoretical versus experimental IR and VCD spectra using different functionals with the 6-31G* basis set for respectively $\mathbf{1}(\mathrm{a}), \mathbf{2}(\mathrm{b})$ and $\mathbf{3}(\mathrm{c})$.

\begin{tabular}{|l|l|l|l|}
\hline \multicolumn{5}{|l|}{ A } \\
\hline functional & $\mathrm{S}_{\mathrm{fg}}$ IR & $\mathrm{S}_{\mathrm{fg}}$ VCD & $\sigma$ \\
\hline B1LYP & 0.956 & 0.833 & 0.954 \\
\hline B3LYP & 0.963 & 0.848 & 0.960 \\
\hline B3P86 & 0.942 & 0.819 & 0.942 \\
\hline B3PW91 & 0.947 & 0.822 & 0.945 \\
\hline B98 & 0.957 & 0.839 & 0.955 \\
\hline BHandH & 0.845 & 0.740 & 0.901 \\
\hline BHandHLYP & 0.882 & 0.750 & 0.906 \\
\hline MPW1PW91 & 0.934 & 0.806 & 0.934 \\
\hline PBE1PBE & 0.933 & 0.808 & 0.932 \\
\hline
\end{tabular}

\begin{tabular}{|l|l|l|l|}
\hline \multicolumn{4}{|l|}{ B } \\
\hline functional & $\mathrm{S}_{\mathrm{fg}}$ IR & $\mathrm{S}_{\mathrm{fg}}$ VCD & $\sigma$ \\
\hline B1LYP & 0.767 & 0.741 & 0.944 \\
\hline B3LYP & 0.768 & 0.765 & 0.952 \\
\hline B3P86 & 0.766 & 0.758 & 0.941 \\
\hline B3PW91 & 0.763 & 0.755 & 0.942 \\
\hline B98 & 0.754 & 0.765 & 0.944 \\
\hline BHandH & 0.707 & 0.651 & 0.900 \\
\hline BHandHLYP & 0.763 & 0.651 & 0.901 \\
\hline MPW1PW91 & 0.767 & 0.743 & 0.931 \\
\hline PBE1PBE & 0.764 & 0.746 & 0.930 \\
\hline
\end{tabular}

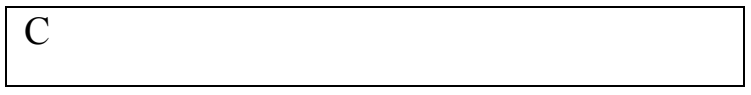




\begin{tabular}{|l|l|l|l|}
\hline functional & $\mathrm{S}_{\mathrm{fg}}$ IR & $\mathrm{S}_{\mathrm{fg}}$ VCD & $\sigma$ \\
\hline B1LYP & 0.930 & 0.823 & 0.967 \\
\hline B3LYP & 0.925 & 0.778 & 0.974 \\
\hline B3P86 & 0.939 & 0.822 & 0.969 \\
\hline B3PW91 & 0.936 & 0.819 & 0.969 \\
\hline B98 & 0.938 & 0.771 & 0.977 \\
\hline BHandH & 0.912 & 0.629 & 0.938 \\
\hline BHandHLYP & 0.906 & 0.648 & 0.930 \\
\hline MPW1PW91 & 0.931 & 0.723 & 0.962 \\
\hline PBE1PBE & 0.937 & 0.735 & 0.963 \\
\hline
\end{tabular}


Assignment of the experimental peaks of (+)-1 based on the B3LYP/6-31G* simulated spectra. The experimental values of the vibrational frequencies $\left(\right.$ in $\left.\mathrm{cm}^{-1}\right)$, dipole $\left(\mathrm{D}\right.$ in $10^{-}$ ${ }^{40} \mathrm{esu}^{2} \mathrm{~cm}^{2}$ ) and rotational strength $\left(\mathrm{R}\right.$ in $10^{-44} \mathrm{esu}^{2} \mathrm{~cm}^{2}$ ) were Lorentzian fitted. Fundamentals are labeled according to the minimum $\left(\mathrm{a} \equiv \mathrm{eGeG}^{\prime} \mathrm{b} \equiv \mathrm{eG}^{\prime} \mathrm{eT}\right)$. Calculated values of $\mathrm{D}$ and $\mathrm{R}$ are Boltzmann weighed according to table 1.

\begin{tabular}{|c|c|c|c|c|c|c|}
\hline \multirow{2}{*}{ Fund } & \multicolumn{3}{|c|}{ Experimental } & \multicolumn{3}{|c|}{ B3LYP/6-31G* } \\
\hline & freq & $\mathrm{D}$ & $\mathrm{R}$ & Freq $^{a}$ & D & $\mathrm{R}$ \\
\hline $34 \mathrm{a}$ & 1024.83 & 104.57 & 14.28 & 1024.48 & 27.07 & 24.45 \\
\hline $34 b$ & 1034.65 & $\perp$ & -12.33 & 1030.40 & 9.83 & -6.09 \\
\hline $35 \mathrm{a}$ & 1042.73 & 201.76 & 116.04 & 1035.31 & 169.35 & 10.25 \\
\hline $35 b$ & & $\perp$ & $\perp$ & 1041.08 & 3.83 & 2.48 \\
\hline $36 \mathrm{~b}$ & 1054.98 & 75.22 & 106.26 & 1045.43 & 15.79 & 14.56 \\
\hline $36 \mathrm{a}$ & 1064.06 & 165.79 & $\perp$ & 1047.04 & 93.08 & 53.82 \\
\hline $37 \mathrm{~b}$ & 1075.09 & 339.49 & -137.13 & 1085.52 & 47.18 & 3.94 \\
\hline $37 \mathrm{a}$ & & $\perp$ & $\perp$ & 1085.62 & 289.06 & -2.34 \\
\hline $38 \mathrm{a}$ & 1130.65 & 17.29 & -28.06 & 1119.84 & 21.77 & -38.96 \\
\hline $38 \mathrm{~b}$ & 1137.29 & 30.95 & | & 1128.28 & 3.87 & -3.02 \\
\hline $39 a$ & & & $\stackrel{\perp}{\perp}$ & 1128.49 & 8.86 & -3.15 \\
\hline $39 b$ & 1139.53 & & 18.17 & 1132.46 & 1.72 & 2.60 \\
\hline $40 \mathrm{a}$ & & $\perp$ & $\perp$ & 1133.15 & 8.04 & 12.59 \\
\hline $40 \mathrm{~b}$ & 1147.58 & 17.66 & -36.21 & 1143.99 & 3.45 & -2.93 \\
\hline $41 b$ & 1165.08 & 28.78 & 15.96 & 1148.67 & 4.14 & 8.63 \\
\hline $41 \mathrm{a}$ & & $\perp$ & $\perp$ & 1151.12 & 7.42 & 11.04 \\
\hline $42 b$ & 1187.64 & 49.24 & 39.94 & 1173.48 & 0.55 & 0.05 \\
\hline $42 a$ & & $\perp$ & $\perp$ & 1174.57 & 1.03 & 1.45 \\
\hline $43 b$ & 1204.16 & 48.13 & 55.55 & 1185.42 & 21.25 & 7.56 \\
\hline $43 a$ & & | & 1 & 1193.64 & 3.87 & 2.47 \\
\hline $44 \mathrm{~b}$ & & $\perp$ & $\perp$ & 1201.03 & 11.38 & 1.46 \\
\hline $44 a$ & 1226.20 & 22.62 & -54.94 & 1218.27 & 41.35 & -2.22 \\
\hline $45 \mathrm{a}$ & 1229.94 & 87.14 & & 1221.88 & 16.76 & -14.89 \\
\hline $45 \mathrm{~b}$ & & & & 1222.81 & 1.96 & -1.82 \\
\hline $46 \mathrm{~b}$ & & & & 1224.50 & 3.00 & -3.35 \\
\hline $46 a$ & & $\dot{\perp}$ & $\dot{\perp}$ & 1231.82 & 3.35 & -5.41 \\
\hline $47 a$ & 1256.29 & 83.32 & -66.89 & 1258.70 & 38.79 & -42.67 \\
\hline $47 \mathrm{~b}$ & & & & 1260.17 & 5.49 & -7.41 \\
\hline $48 \mathrm{~b}$ & & & & 1272.35 & 0.77 & -2.19 \\
\hline $48 \mathrm{a}$ & & $\dot{\perp}$ & $\perp$ & 1275.07 & 16.58 & -10.49 \\
\hline $49 a$ & 1280.89 & 27.34 & 24.52 & 1279.92 & 21.74 & 13.33 \\
\hline $49 b$ & & $\perp$ & $\perp$ & 1280.69 & 0.68 & 0.59 \\
\hline $50 \mathrm{~b}$ & 1288.31 & 57.44 & -47.59 & 1289.41 & 1.04 & -2.47 \\
\hline $50 \mathrm{a}$ & & & $\perp$ & 1289.77 & 19.59 & -8.56 \\
\hline $51 \mathrm{a}$ & 1295.42 & $\perp$ & 13.93 & 1296.22 & 7.77 & 4.69 \\
\hline $51 \mathrm{~b}$ & 1304.38 & 66.34 & & 1300.70 & 4.95 & -0.85 \\
\hline $52 \mathrm{a}$ & & & & 1304.39 & 19.61 & 2.72 \\
\hline $52 \mathrm{~b}$ & & $\perp$ & $\perp$ & 1311.45 & 6.36 & -1.91 \\
\hline $53 a$ & 1325.15 & 133.79 & 93.86 & 1328.19 & 225.14 & 20.39 \\
\hline $53 b$ & 1334.61 & 92.61 & & 1331.55 & 44.68 & 0.58 \\
\hline $54 a$ & & & & 1340.82 & 19.91 & -1.80 \\
\hline
\end{tabular}




\begin{tabular}{|c|c|c|c|c|c|c|}
\hline $54 b$ & & $\perp$ & | & 1344.28 & 0.32 & -0.05 \\
\hline $55 b$ & 1379.26 & 46.53 & $\perp$ & 1383.32 & 6.76 & -3.17 \\
\hline $55 \mathrm{a}$ & 1388.30 & 115.43 & -66.80 & 1391.64 & 167.52 & -28.64 \\
\hline $56 a$ & 1388.85 & $\perp$ & -84.63 & 1409.11 & 42.26 & -29.47 \\
\hline $56 b$ & 1402.34 & 52.32 & & 1416.98 & 6.86 & -3.94 \\
\hline $57 \mathrm{a}$ & 1420.35 & 13.13 & & 1434.65 & 1.81 & -2.65 \\
\hline $57 b$ & & $\perp$ & $\perp$ & 1439.29 & 0.61 & -0.63 \\
\hline $58 \mathrm{a}$ & 1438.23 & 45.75 & 4.62 & 1455.09 & 41.22 & 15.18 \\
\hline $58 \mathrm{~b}$ & & $\perp$ & $\perp$ & 1456.28 & 7.83 & 3.45 \\
\hline $59 a$ & 1453.52 & 78.36 & -28.40 & 1465.29 & 1.44 & -1.73 \\
\hline $59 b$ & & $\perp$ & $\perp$ & 1466.48 & 0.31 & -0.45 \\
\hline $60 a$ & 1468.36 & 85.65 & 29.74 & 1469.27 & 35.92 & 16.20 \\
\hline $\begin{array}{l}60 \mathrm{~b} \\
{ }^{\mathrm{a}} \mathrm{sca}\end{array}$ & actor 0.96 & $\perp$ & $\perp$ & 1469.87 & 5.79 & 2.66 \\
\hline
\end{tabular}


Assignment of the experimental peaks of (+)-1 based on the B3LYP/cc-pVTZ simulated spectra. The experimental values of the vibrational frequencies (in $\mathrm{cm}^{-1}$ ), dipole (D in $10^{-}$ $\left.{ }^{40} \mathrm{esu}^{2} \mathrm{~cm}^{2}\right)$ and rotational strength $\left(\mathrm{R}\right.$ in $10^{-44} \mathrm{esu}^{2} \mathrm{~cm}^{2}$ ) were Lorentzian fitted. Fundamentals are labeled according to the minimum $\left(\mathrm{a} \equiv \mathrm{eGeG}^{\prime} \mathrm{b} \equiv \mathrm{eG}^{\prime} \mathrm{eT}\right)$. Calculated values of $\mathrm{D}$ and $\mathrm{R}$ are Boltzmann weighed according to table 1 .

\begin{tabular}{|c|c|c|c|c|c|c|}
\hline \multirow{2}{*}{ Fund } & \multicolumn{2}{|c|}{ Experimental } & & \multicolumn{3}{|c|}{ B3LYP/cc-pVTZ } \\
\hline & freq & $\mathrm{D}$ & $\mathrm{R}$ & Freq $^{\mathrm{a}}$ & $\mathrm{D}$ & $\mathrm{R}$ \\
\hline $34 \mathrm{a}$ & 1024.83 & 104.57 & 14.28 & 1025.47 & 36.22 & 34.00 \\
\hline $34 b$ & 1034.65 & $\perp$ & -12.33 & 1031.87 & 21.37 & -10.81 \\
\hline $35 \mathrm{a}$ & 1042.73 & 201.76 & 116.04 & 1036.53 & 186.82 & 17.44 \\
\hline $35 b$ & & $\perp$ & $\perp$ & 1039.70 & 18.94 & 15.34 \\
\hline $36 a$ & 1054.98 & 75.22 & 106.26 & 1047.37 & 49.35 & 24.85 \\
\hline $36 b$ & 1064.06 & 165.79 & $\perp$ & 1046.22 & 13.71 & 11.46 \\
\hline $37 \mathrm{a}$ & 1075.09 & 339.49 & -137.13 & 1076.72 & 272.24 & -9.80 \\
\hline $37 b$ & & $\perp$ & $\perp$ & 1076.68 & 75.90 & 4.05 \\
\hline $38 \mathrm{a}$ & 1130.65 & 17.29 & -28.06 & 1123.10 & 18.43 & -31.33 \\
\hline $38 b$ & 1137.29 & 30.95 & & 1132.21 & 3.91 & -3.48 \\
\hline $39 a$ & & & $\perp$ & 1132.26 & 5.28 & 3.95 \\
\hline $39 b$ & 1139.53 & & 18.17 & 1135.90 & 5.87 & 4.76 \\
\hline $40 \mathrm{a}$ & & $\perp$ & $\perp$ & 1136.54 & 12.24 & 11.26 \\
\hline $40 \mathrm{~b}$ & 1147.58 & 17.66 & -36.21 & 1147.10 & 6.51 & -2.79 \\
\hline $41 \mathrm{a}$ & 1165.08 & 28.78 & 15.96 & 1157.47 & 8.23 & 4.92 \\
\hline $41 \mathrm{~b}$ & & $\perp$ & $\perp$ & 1155.84 & 4.57 & 10.55 \\
\hline $42 a$ & 1187.64 & 49.24 & 39.94 & 1178.80 & 0.83 & 1.62 \\
\hline $42 b$ & & $\perp$ & $\perp$ & 1177.42 & 1.96 & 0.05 \\
\hline $43 a$ & 1204.16 & 48.13 & 55.55 & 1202.88 & 4.60 & 0.97 \\
\hline $43 b$ & & & & 1187.34 & 36.87 & 11.25 \\
\hline $44 a$ & & $\perp$ & $\perp$ & 1221.37 & 28.76 & 1.89 \\
\hline $44 b$ & 1226.20 & 22.62 & -54.94 & 1206.79 & 4.52 & 0.59 \\
\hline $45 a$ & 1229.94 & 87.14 & & 1226.62 & 20.47 & 1.43 \\
\hline $45 b$ & & & & 1225.48 & 0.37 & -0.10 \\
\hline $46 a$ & & & & 1233.94 & 2.84 & -15.22 \\
\hline $46 b$ & & $\perp$ & $\perp$ & 1229.96 & 6.47 & -7.15 \\
\hline $47 a$ & 1256.29 & 83.32 & -66.89 & 1254.62 & 45.35 & -43.09 \\
\hline $47 b$ & & & & 1256.42 & 11.13 & -12.73 \\
\hline $48 a$ & & & & 1273.32 & 9.86 & -7.34 \\
\hline $48 b$ & & $\dot{\perp}$ & $\dot{\perp}$ & 1270.10 & 0.51 & -1.21 \\
\hline $49 a$ & 1280.89 & 27.34 & 24.52 & 1280.17 & 5.27 & 5.19 \\
\hline $49 b$ & & $\perp$ & $\perp$ & 1280.15 & 1.49 & 0.47 \\
\hline $50 \mathrm{a}$ & 1288.31 & 57.44 & -47.59 & 1288.46 & 24.34 & -3.91 \\
\hline $50 \mathrm{~b}$ & & & $\perp$ & 1286.72 & 3.55 & -6.84 \\
\hline $51 \mathrm{a}$ & 1295.42 & $\perp$ & 13.93 & 1294.30 & 6.87 & 7.23 \\
\hline $51 \mathrm{~b}$ & 1304.38 & 66.34 & & 1303.29 & 10.18 & -2.06 \\
\hline $52 \mathrm{a}$ & & & & 1305.18 & 33.93 & 3.08 \\
\hline $52 b$ & & $\perp$ & $\perp$ & 1310.74 & 2.48 & 0.94 \\
\hline $53 a$ & 1325.15 & 133.79 & 93.86 & 1321.09 & 144.19 & 30.54 \\
\hline $53 b$ & 1334.61 & 92.61 & & 1324.16 & 55.26 & 3.66 \\
\hline $54 \mathrm{a}$ & & & & 1336.45 & 14.25 & -1.96 \\
\hline $54 \mathrm{~b}$ & & $\perp$ & & 1340.64 & 0.41 & -0.15 \\
\hline $55 \mathrm{a}$ & 1379.26 & 46.53 & $\perp$ & 1386.73 & 101.65 & -16.15 \\
\hline
\end{tabular}




$\begin{array}{ccrrrrr}55 \mathrm{~b} & 1388.30 & 115.43 & -66.80 & 1377.96 & 8.00 & -5.45 \\ 56 \mathrm{a} & 1388.85 & \perp & -84.63 & 1396.90 & 49.00 & -40.48 \\ 56 \mathrm{~b} & 1402.34 & 52.32 & \mid & 1402.41 & 7.58 & -4.98 \\ 57 \mathrm{a} & 1420.35 & 13.13 & \perp & 1435.25 & 0.64 & -0.84 \\ 57 \mathrm{~b} & & \perp & \perp & 1439.14 & 0.43 & -0.36 \\ 58 \mathrm{a} & 1438.23 & 45.75 & 4.62 & 1450.43 & 21.96 & 2.65 \\ 58 \mathrm{~b} & & \perp & \perp & 1451.00 & 6.38 & 1.11 \\ 59 \mathrm{a} & 1453.52 & 78.36 & -28.40 & 1457.06 & 4.35 & -5.04 \\ 59 \mathrm{~b} & & \perp & \perp & 1458.40 & 1.27 & -1.78 \\ 60 \mathrm{a} & 1468.36 & 85.65 & 29.74 & 1465.85 & 33.80 & 10.81 \\ 60 \mathrm{~b} & & \mid & & 1466.50 & 4.57 & 1.98 \\ 61 \mathrm{a} & & & & 1466.66 & 0.69 & -2.05 \\ 61 \mathrm{~b} & & & & 1467.18 & 3.37 & 0.11 \\ 62 \mathrm{a} & & & & 1468.43 & 26.00 & 8.36 \\ 62 \mathrm{~b} & & \perp & \perp & 1468.90 & 9.68 & 3.04\end{array}$

Correlation Coefficients - D: 0.45 / R: 0.30 
Assignment of the experimental peaks of (+)-2 based on the B3LYP/6-31G* simulated spectra. The experimental values of the vibrational frequencies $\left(\right.$ in $\left.\mathrm{cm}^{-1}\right)$, dipole $\left(\mathrm{D}\right.$ in $10^{-}$ ${ }^{40} \mathrm{esu}^{2} \mathrm{~cm}^{2}$ ) and rotational strength ( $\mathrm{R}$ in $10^{-44} \mathrm{esu}^{2} \mathrm{~cm}^{2}$ ) were Lorentzian fitted. The fundamentals listed are of minimum $\mathrm{eG}^{\text {'. }}$

\begin{tabular}{|c|c|c|c|c|c|c|}
\hline \multirow{2}{*}{ Fund } & \multicolumn{3}{|c|}{ experimental } & \multicolumn{3}{|c|}{ B3LYP/6-31G* } \\
\hline & freq & $\mathrm{D}$ & $\mathrm{R}$ & Freq $^{a}$ & $\mathrm{D}$ & $\mathrm{R}$ \\
\hline 34 & 1068.74 & 296.57 & 222.04 & 1069.24 & 89.45 & 28.21 \\
\hline 35 & 1092.45 & 160.69 & -33.90 & 1089.67 & 252.24 & 79.33 \\
\hline 36 & 1137.94 & 25.48 & -12.99 & 1132.22 & 1.71 & -18.45 \\
\hline 37 & 1147.67 & 32.92 & 27.85 & 1134.61 & 7.54 & 14.06 \\
\hline 38 & & $\perp$ & $\perp$ & 1138.62 & 24.39 & 19.05 \\
\hline 39 & 1179.41 & 49.47 & -42.38 & 1168.36 & 43.47 & -22.67 \\
\hline 40 & 1194.59 & 57.49 & 15.10 & 1190.49 & 41.42 & 11.15 \\
\hline 41 & 1213.49 & 53.64 & 2.13 & 1206.76 & 0.52 & 5.33 \\
\hline 42 & & $\perp$ & $\perp$ & 1212.71 & 11.34 & 9.29 \\
\hline 43 & 1235.08 & 72.54 & -111.60 & 1227.28 & 108.41 & -42.98 \\
\hline 44 & 1249.79 & 104.86 & 38.00 & 1247.91 & 60.56 & 28.66 \\
\hline 45 & 1257.26 & 30.37 & -48.84 & 1257.10 & 100.35 & -74.32 \\
\hline & 1259.64 & 73.19 & $\perp$ & & $\perp$ & $\perp$ \\
\hline 46 & 1278.90 & 125.80 & -65.43 & 1277.09 & 131.08 & -6.35 \\
\hline 47 & 1286.09 & 78.04 & -18.54 & 1284.74 & 13.93 & -16.92 \\
\hline 48 & 1299.15 & 83.38 & -2.72 & 1298.52 & 13.48 & -3.19 \\
\hline 49 & 1331.65 & 149.02 & 84.48 & 1332.26 & 214.69 & 21.33 \\
\hline 50 & 1341.54 & 44.48 & -32.00 & 1345.75 & 11.97 & -23.31 \\
\hline 51 & 1390.98 & 55.57 & 6.17 & 1409.29 & 93.06 & 4.86 \\
\hline 52 & 1406.57 & 86.00 & -11.84 & 1426.98 & 93.42 & -10.57 \\
\hline 53 & 1416.32 & 41.12 & -6.10 & 1437.65 & 34.33 & -3.52 \\
\hline & 1427.01 & $\perp$ & 2.09 & & $\perp$ & $\perp$ \\
\hline 54 & 1442.73 & 57.15 & 6.53 & 1456.68 & 78.81 & 24.64 \\
\hline 55 & 1438.93 & 70.21 & $\perp$ & 1466.39 & 43.45 & 7.41 \\
\hline 56 & 1454.00 & 74.69 & -10.08 & 1472.05 & 14.76 & 9.22 \\
\hline 57 & 1473.41 & 116.00 & 23.56 & 1477.54 & 7.52 & -3.16 \\
\hline 58 & 1586.97 & 104.35 & - & 1583.22 & 66.62 & -1.67 \\
\hline 59 & 1613.42 & 154.70 & -17.25 & 1601.36 & 86.44 & -1.50 \\
\hline 60 & 1686.50 & 1215.25 & 151.90 & 1713.72 & 608.98 & 27.80 \\
\hline
\end{tabular}

${ }^{\mathrm{a}}$ scaled with factor 0.967

Correlation Coefficients - D: 0.72 / R: 0.47 
Assignment of the experimental peaks of (+)-2 based on the B3LYP/cc-pVTZ simulated spectra. The experimental values of the vibrational frequencies $\left(\right.$ in $\left.\mathrm{cm}^{-1}\right)$, dipole (D in $10^{-}$ ${ }^{40} \mathrm{esu}^{2} \mathrm{~cm}^{2}$ ) and rotational strength ( $\mathrm{R}$ in $10^{-44} \mathrm{esu}^{2} \mathrm{~cm}^{2}$ ) were Lorentzian fitted. The fundamentals listed are of minimum eG'.

\begin{tabular}{|c|c|c|c|c|c|c|}
\hline \multirow{2}{*}{ Fund } & \multicolumn{3}{|c|}{ experimental } & \multicolumn{3}{|c|}{ B3LYP/cc-pVTZ } \\
\hline & freq & $\mathrm{D}$ & $\mathrm{R}$ & Freq $^{a}$ & $\mathrm{D}$ & $\mathrm{R}$ \\
\hline 33 & 1026.20 & 66.25 & -103.77 & 1023.92 & 68.95 & -29.65 \\
\hline 34 & 1068.74 & 296.57 & 222.04 & 1075.34 & 97.67 & 71.77 \\
\hline 35 & 1092.45 & 160.69 & -33.90 & 1086.02 & 264.70 & 36.88 \\
\hline 36 & 1137.94 & 25.48 & -12.99 & 1135.91 & 0.89 & -11.24 \\
\hline 37 & 1147.67 & 32.92 & 27.85 & 1139.12 & 3.48 & 4.59 \\
\hline 38 & & $\perp$ & $\perp$ & 1144.39 & 31.47 & 20.78 \\
\hline 39 & 1179.41 & 49.47 & -42.38 & 1172.38 & 46.84 & -23.81 \\
\hline 40 & 1194.59 & 57.49 & 15.10 & 1193.96 & 40.42 & 12.98 \\
\hline 41 & 1213.49 & 53.64 & 2.13 & 1212.66 & 0.32 & 6.13 \\
\hline 42 & & $\perp$ & $\perp$ & 1218.65 & 5.95 & 0.41 \\
\hline 43 & 1235.08 & 72.54 & -111.60 & 1232.60 & 92.22 & -46.70 \\
\hline 44 & 1249.79 & 104.86 & 38.00 & 1247.34 & 92.79 & 38.55 \\
\hline \multirow[t]{2}{*}{45} & 1257.26 & 30.37 & -48.84 & 1256.37 & 92.25 & -70.55 \\
\hline & 1259.64 & 73.19 & $\perp$ & & $\perp$ & $\perp$ \\
\hline 46 & 1278.90 & 125.80 & -65.43 & 1277.02 & 102.59 & -11.25 \\
\hline 47 & 1286.09 & 78.04 & -18.54 & 1283.63 & 23.51 & -23.90 \\
\hline 48 & 1299.15 & 83.38 & -2.72 & 1301.51 & 11.16 & -6.08 \\
\hline 49 & 1331.65 & 149.02 & 84.48 & 1328.97 & 168.95 & 25.48 \\
\hline 50 & 1341.54 & 44.48 & -32.00 & 1341.69 & 11.04 & -21.88 \\
\hline 51 & 1390.98 & 55.57 & 6.17 & 1402.88 & 106.45 & 10.34 \\
\hline 52 & 1406.57 & 86.00 & -11.84 & 1418.96 & 42.00 & -7.12 \\
\hline \multirow[t]{2}{*}{53} & 1416.32 & 41.12 & -6.10 & 1434.56 & 61.77 & 0.84 \\
\hline & 1427.01 & $\perp$ & 2.09 & & $\perp$ & $\perp$ \\
\hline 54 & 1442.73 & 57.15 & 6.53 & 1451.52 & 46.27 & 9.61 \\
\hline 55 & 1438.93 & 70.21 & $\perp$ & 1457.56 & 34.08 & 0.18 \\
\hline 56 & 1454.00 & 74.69 & -10.08 & 1468.08 & 2.39 & -5.60 \\
\hline 57 & 1473.41 & 116.00 & 23.56 & 1471.41 & 70.55 & 23.85 \\
\hline 58 & 1586.97 & 104.35 & - & 1586.07 & 71.63 & -0.55 \\
\hline 59 & 1613.42 & 154.70 & -17.25 & 1603.03 & 90.97 & 1.47 \\
\hline 60 & 1686.50 & 1215.25 & 151.90 & 1707.75 & 670.62 & 35.77 \\
\hline wit & or 0.977 & & & & & \\
\hline
\end{tabular}


Assignment of the experimental peaks of $(-)_{356}-3$ based on the B3LYP/6-31G* simulated spectra. The experimental values of the vibrational frequencies $\left(\right.$ in $\left.\mathrm{cm}^{-1}\right)$, dipole (D in $10^{-}$ ${ }^{40} \mathrm{esu}^{2} \mathrm{~cm}^{2}$ ) and rotational strength $\left(\mathrm{R}\right.$ in $\left.10^{-44} \mathrm{esu}^{2} \mathrm{~cm}^{2}\right)$ were Lorentzian fitted.

\begin{tabular}{ccrrrrr} 
Fund & \multicolumn{3}{c}{ experimental } & \multicolumn{3}{c}{ B3LYP/6-31G* } \\
34 & freq & $\mathrm{D}$ & $\mathrm{R}$ & Freq $^{\mathrm{a}}$ & $\mathrm{D}$ & \multicolumn{1}{c}{$\mathrm{R}$} \\
35 & 1088.34 & 9.94 & -7.91 & 1066.00 & 2.47 & -6.54 \\
36 & 1131.78 & 17.90 & 5.81 & 1126.37 & 10.51 & -3.41 \\
37 & 1165.18 & 7.28 & 43.73 & 1162.92 & 12.36 & -1.15 \\
38 & 1167.03 & 28.66 & $\perp$ & 1163.87 & 2.51 & 4.88 \\
39 & 1194.46 & 6.46 & 38.53 & 1180.22 & 0.96 & 1.96 \\
40 & 1207.20 & 50.50 & -56.82 & 1198.71 & 18.71 & -15.41 \\
41 & & $\perp$ & $\perp$ & 1204.84 & 14.03 & -22.18 \\
42 & 1228.58 & 23.08 & 31.82 & 1219.22 & 13.94 & 23.09 \\
43 & 1232.30 & 68.18 & -49.03 & 1223.08 & 25.13 & -38.50 \\
44 & 1248.24 & 17.30 & 9.20 & 1243.04 & 24.79 & 0.19 \\
45 & 1282.52 & 20.61 & 3.67 & 1279.92 & 4.67 & 4.14 \\
46 & 1288.05 & $\perp$ & 22.77 & 1280.34 & 3.55 & 8.13 \\
47 & 1327.72 & 16.19 & 2.83 & 1312.62 & 6.76 & 6.66 \\
48 & 1351.85 & 18.10 & -17.56 & 1338.69 & 1.25 & -5.94 \\
49 & 1363.04 & 7.60 & 32.49 & 1376.14 & 25.68 & -32.90 \\
50 & 1367.16 & 45.17 & 41.95 & 1376.29 & 44.42 & 78.25 \\
51 & 1422.65 & 3.87 & 33.84 & 1441.44 & 9.48 & -6.93 \\
52 & 1431.12 & 25.48 & 20.00 & 1441.67 & 27.21 & 9.54 \\
53 & 1454.67 & 31.83 & 11.82 & 1453.16 & 2.19 & 2.51 \\
54 & 1455.85 & $\perp$ & 3.32 & 1454.53 & 31.83 & 15.33 \\
& 1459.12 & $\perp$ & 25.65 & & $\perp$ & $\perp$ \\
55 & 1601.74 & 23.52 & -10.89 & 1590.68 & 0.44 & -3.94 \\
56 & 1650.93 & 21.00 & -5.35 & 1620.19 & 0.63 & -1.33 \\
a & & & & & \\
Correlation Coefficients - D: & $0.79 / \mathrm{R}: 0.23$ & & & &
\end{tabular}


Assignment of the experimental peaks of $(-)_{356}-3$ based on the B3LYP/cc-pVTZ simulated spectra. The experimental values of the vibrational frequencies $\left(\right.$ in $\left.\mathrm{cm}^{-1}\right)$, dipole ( $\mathrm{D}$ in $10^{-}$ ${ }^{40} \mathrm{esu}^{2} \mathrm{~cm}^{2}$ ) and rotational strength $\left(\mathrm{R}\right.$ in $\left.10^{-44} \mathrm{esu}^{2} \mathrm{~cm}^{2}\right)$ were Lorentzian fitted.

\begin{tabular}{|c|c|c|c|c|c|c|}
\hline \multirow{2}{*}{ Fund } & \multicolumn{3}{|c|}{ experimental } & \multicolumn{3}{|c|}{ B3LYP/cc-pVTZ } \\
\hline & freq & $\mathrm{D}$ & $\mathrm{R}$ & Freq $^{a}$ & $\mathrm{D}$ & $\mathrm{R}$ \\
\hline 33 & 1037.20 & 95.81 & -18.23 & 1031.95 & 74.06 & -18.84 \\
\hline 34 & 1088.34 & 9.94 & -7.91 & 1080.92 & 2.76 & -6.81 \\
\hline 35 & & $\perp$ & $\perp$ & 1085.69 & 1.77 & -0.74 \\
\hline 36 & 1131.78 & 17.90 & 5.81 & 1130.17 & 12.13 & 0.01 \\
\hline 37 & 1165.18 & 7.28 & 43.73 & 1166.93 & 11.23 & 3.20 \\
\hline 38 & 1167.03 & 28.66 & $\perp$ & 1167.35 & 3.85 & 7.51 \\
\hline 39 & 1194.46 & 6.46 & 38.53 & 1190.18 & 1.96 & 3.50 \\
\hline 40 & 1207.20 & 50.50 & -56.82 & 1202.51 & 20.32 & -3.33 \\
\hline 41 & & $\perp$ & $\perp$ & 1207.05 & 18.27 & -29.84 \\
\hline 42 & 1228.58 & 23.08 & 31.82 & 1222.13 & 7.75 & 19.53 \\
\hline 43 & 1232.30 & 68.18 & -49.03 & 1228.94 & 26.90 & -45.83 \\
\hline 44 & 1248.24 & 17.30 & 9.20 & 1240.69 & 28.86 & 4.58 \\
\hline 45 & 1282.52 & 20.61 & 3.67 & 1279.20 & 8.49 & 6.33 \\
\hline 46 & 1288.05 & $\perp$ & 22.77 & 1279.67 & 0.86 & 7.23 \\
\hline 47 & 1327.72 & 16.19 & 2.83 & 1314.45 & 4.42 & 5.23 \\
\hline 48 & 1351.85 & 18.10 & -17.56 & 1334.33 & 1.85 & -7.17 \\
\hline 49 & 1363.04 & 7.60 & 32.49 & 1369.96 & 41.21 & 65.74 \\
\hline 50 & 1367.16 & 45.17 & 41.95 & 1371.94 & 20.96 & -28.85 \\
\hline 51 & 1422.65 & 3.87 & 33.84 & 1431.08 & 17.38 & -3.13 \\
\hline 52 & 1431.12 & 25.48 & 20.00 & 1431.33 & 23.68 & 5.64 \\
\hline 53 & 1454.67 & 31.83 & 11.82 & 1452.46 & 0.73 & 1.88 \\
\hline 54 & 1455.85 & & 3.32 & 1456.42 & 43.03 & 20.82 \\
\hline & 1459.12 & $\perp$ & 25.65 & & $\perp$ & $\perp$ \\
\hline 55 & 1601.74 & 23.52 & -10.89 & 1593.71 & 1.00 & -4.67 \\
\hline 56 & 1650.93 & 21.00 & -5.35 & 1622.18 & 0.59 & -1.27 \\
\hline d wit & or 0.977 & & & & & \\
\hline
\end{tabular}




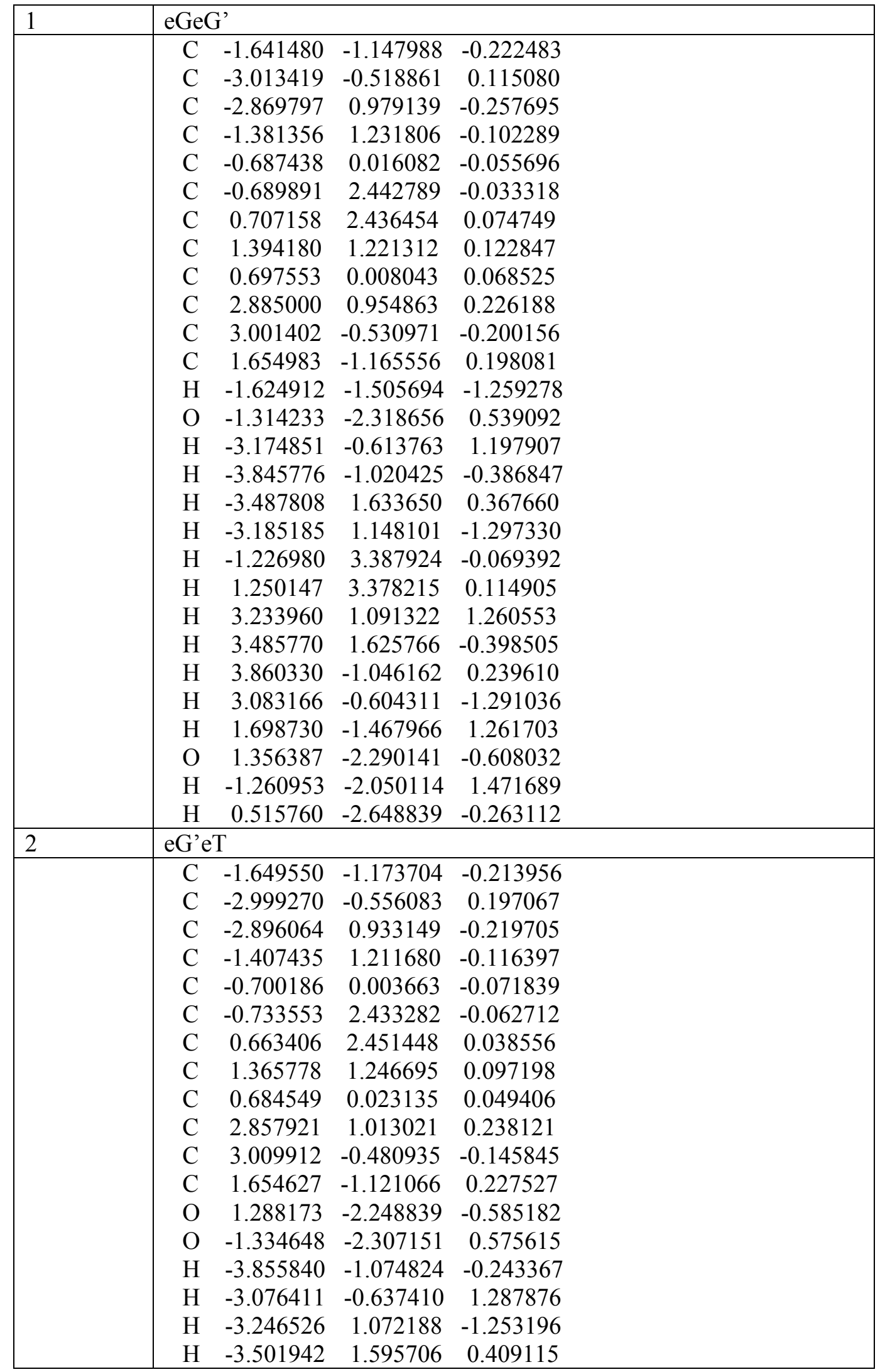




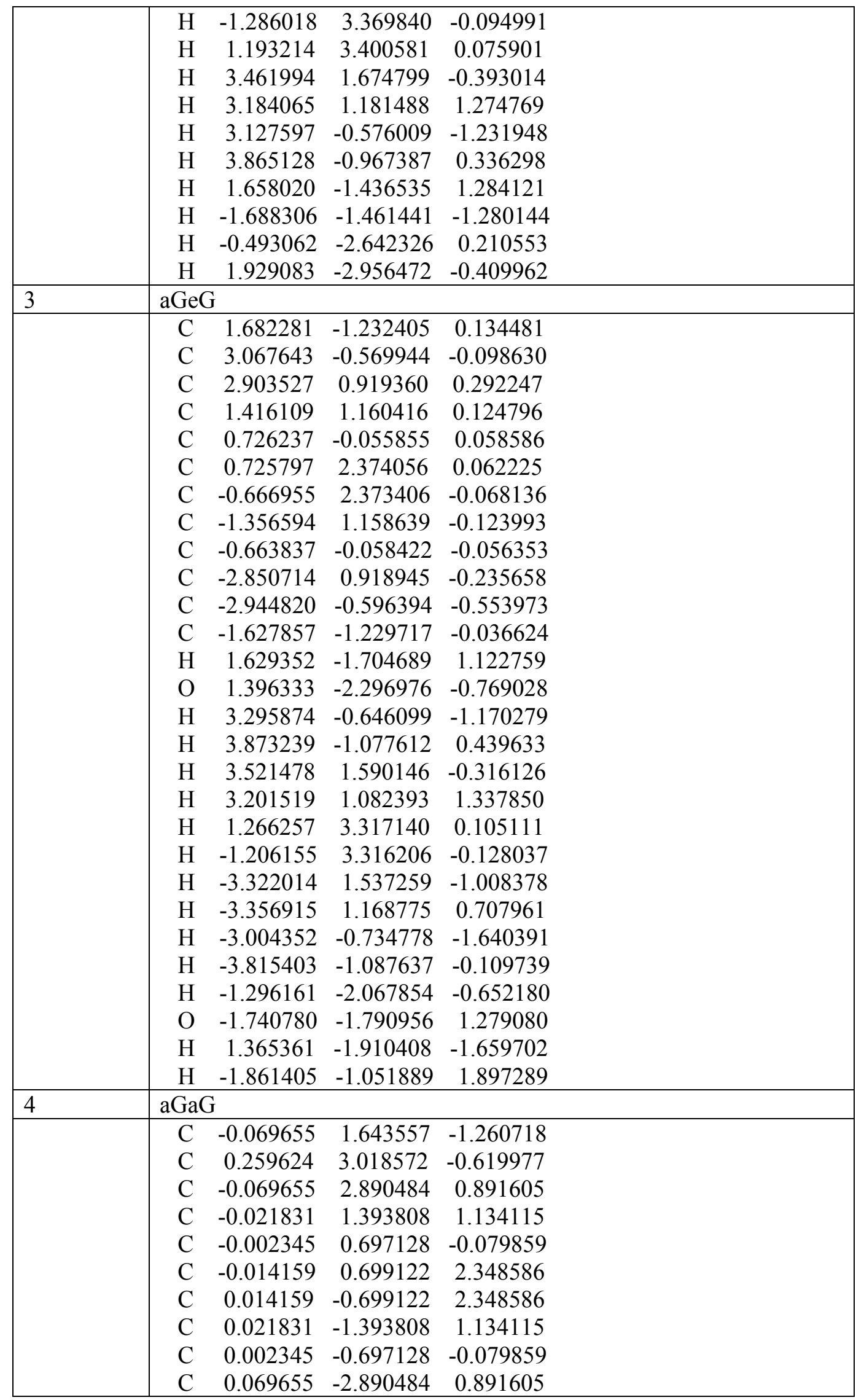




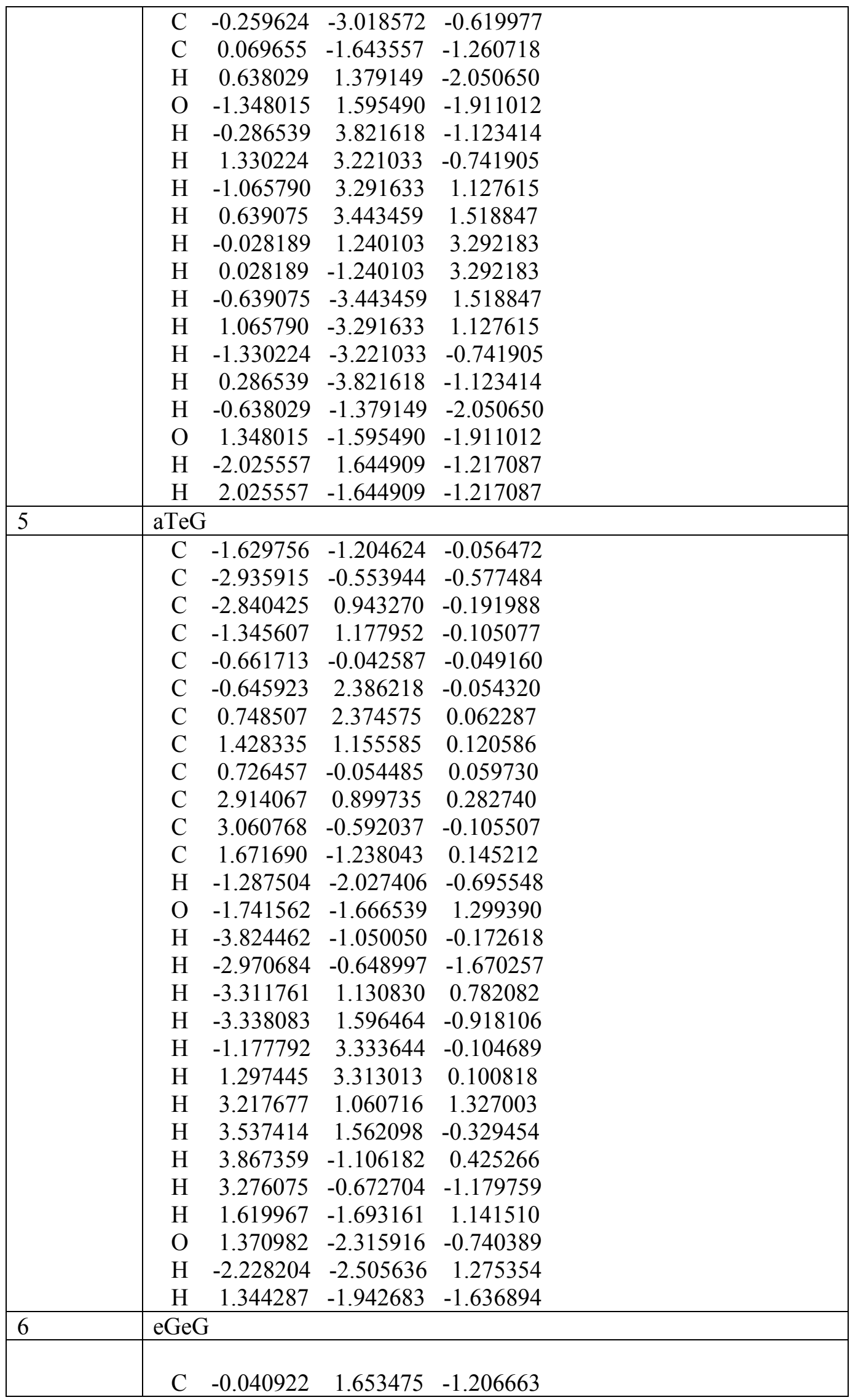




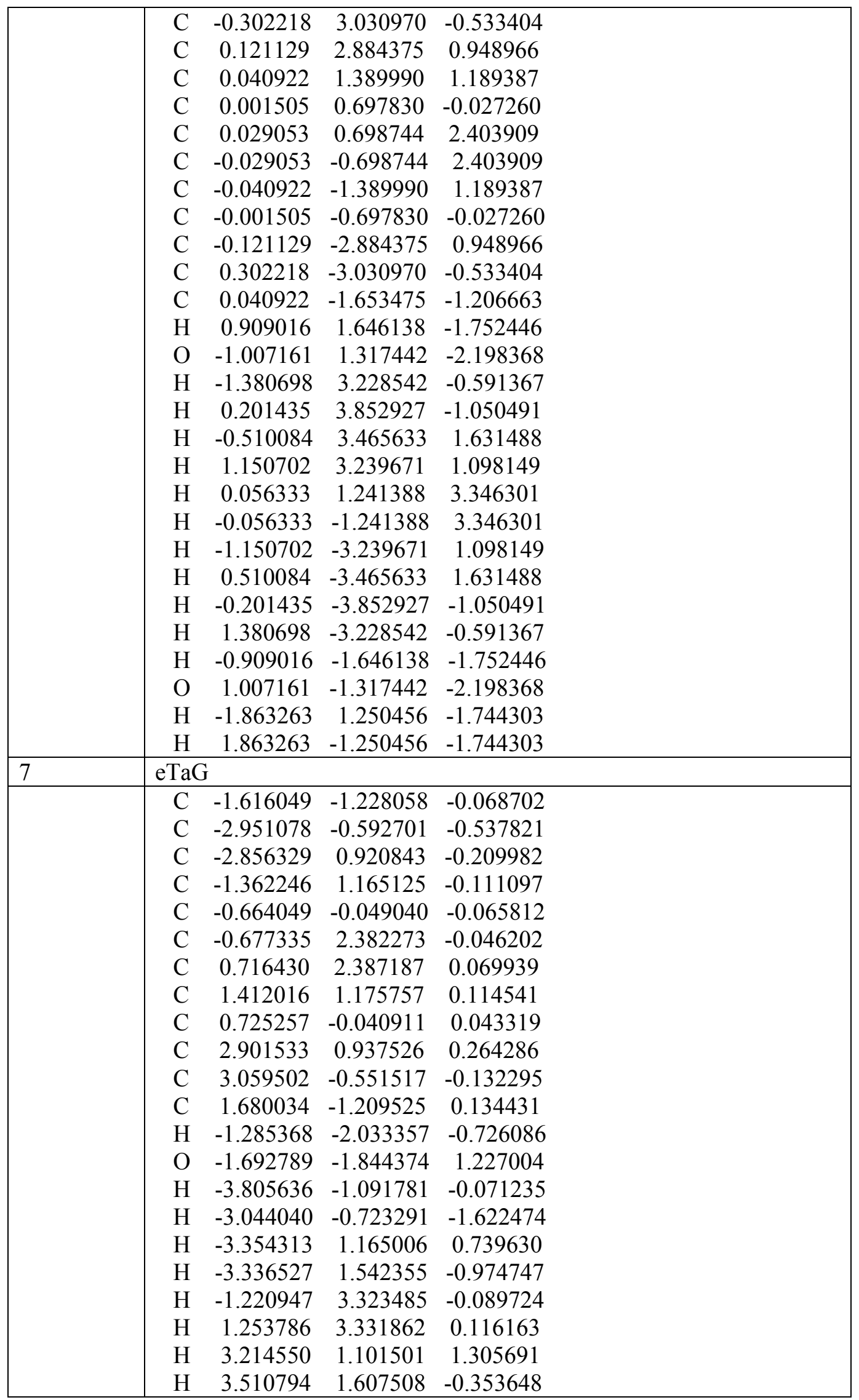




\begin{tabular}{|c|c|c|c|c|}
\hline & $\begin{array}{l}\mathrm{H} \\
\mathrm{H} \\
\mathrm{H} \\
\mathrm{O} \\
\mathrm{H} \\
\mathrm{H}\end{array}$ & $\begin{array}{r}3.883142 \\
3.241064 \\
1.636630 \\
1.340123 \\
-1.825783 \\
1.747859\end{array}$ & $\begin{array}{l}-1.051689 \\
-0.632824 \\
-1.637814 \\
-2.218472 \\
-1.132374 \\
-3.047582\end{array}$ & $\begin{array}{r}0.388318 \\
-1.210005 \\
1.148982 \\
-0.817780 \\
1.873899 \\
-0.526194\end{array}$ \\
\hline 8 & \multicolumn{4}{|c|}{ aGaG' } \\
\hline & $\begin{array}{l}\mathrm{C} \\
\mathrm{C} \\
\mathrm{C} \\
\mathrm{C} \\
\mathrm{C} \\
\mathrm{C} \\
\mathrm{C} \\
\mathrm{C} \\
\mathrm{C} \\
\mathrm{C} \\
\mathrm{C} \\
\mathrm{C} \\
\mathrm{H} \\
\mathrm{O} \\
\mathrm{H} \\
\mathrm{H} \\
\mathrm{H} \\
\mathrm{H} \\
\mathrm{H} \\
\mathrm{H} \\
\mathrm{H} \\
\mathrm{H} \\
\mathrm{H} \\
\mathrm{H} \\
\mathrm{H} \\
\mathrm{O} \\
\mathrm{H} \\
\mathrm{H} \\
\end{array}$ & $\begin{array}{r}1.661547 \\
2.946775 \\
2.866263 \\
1.373757 \\
0.686024 \\
0.674952 \\
-0.722382 \\
-1.407769 \\
-0.704334 \\
-2.903578 \\
-3.010925 \\
-1.655636 \\
1.308900 \\
1.953263 \\
3.834127 \\
2.947522 \\
3.344069 \\
3.364658 \\
1.209463 \\
-1.268690 \\
-3.411746 \\
-3.366848 \\
-3.154255 \\
-3.840736 \\
-1.368821 \\
-1.679783 \\
1.112208 \\
-1.725853 \\
\end{array}$ & $\begin{array}{r}-1.248730 \\
-0.598819 \\
0.890914 \\
1.134308 \\
-0.085557 \\
2.343086 \\
2.336185 \\
1.119887 \\
-0.093154 \\
0.875212 \\
-0.635538 \\
-1.274644 \\
-2.081595 \\
-1.720162 \\
-1.114320 \\
-0.674298 \\
1.048596 \\
1.557886 \\
3.289460 \\
3.276627 \\
1.500105 \\
1.112208 \\
-0.760102 \\
-1.141082 \\
-2.066121 \\
-1.942197 \\
-1.972210 \\
-1.255185 \\
\end{array}$ & $\begin{array}{c}0.046137 \\
0.591301 \\
0.172106 \\
0.075464 \\
0.024231 \\
0.036596 \\
-0.045387 \\
-0.097802 \\
-0.076525 \\
-0.144614 \\
-0.478244 \\
-0.072051 \\
0.669315 \\
-1.280695 \\
0.213831 \\
1.684995 \\
-0.803982 \\
0.884792 \\
0.078717 \\
-0.061166 \\
-0.888052 \\
0.823788 \\
-1.558097 \\
0.024087 \\
-0.773136 \\
1.196833 \\
-1.692974 \\
1.881507 \\
\end{array}$ \\
\hline 9 & \multicolumn{4}{|c|}{$\mathrm{aTaG}$} \\
\hline & $\begin{array}{l}\mathrm{C} \\
\mathrm{C} \\
\mathrm{C} \\
\mathrm{C} \\
\mathrm{C} \\
\mathrm{C} \\
\mathrm{C} \\
\mathrm{C} \\
\mathrm{C} \\
\mathrm{C} \\
\mathrm{C} \\
\mathrm{C} \\
\mathrm{H} \\
\mathrm{O}\end{array}$ & $\begin{array}{c}-1.634092 \\
-2.990324 \\
-2.898873 \\
-1.404904 \\
-0.697686 \\
-0.722053 \\
0.675146 \\
1.377632 \\
0.690746 \\
2.873171 \\
2.973100 \\
1.646539 \\
-1.312870 \\
-1.667508 \\
\end{array}$ & $\begin{array}{r}-1.269014 \\
-0.637474 \\
0.873187 \\
1.128569 \\
-0.079610 \\
2.348023 \\
2.359655 \\
1.151361 \\
-0.066213 \\
0.912775 \\
-0.578909 \\
-1.235823 \\
-2.042090 \\
-1.945511 \\
\end{array}$ & $\begin{array}{c}-0.076948 \\
-0.491793 \\
-0.146839 \\
-0.084703 \\
-0.059249 \\
-0.037278 \\
0.033497 \\
0.071799 \\
0.040085 \\
0.125471 \\
0.533550 \\
0.070764 \\
-0.779796 \\
1.189778 \\
\end{array}$ \\
\hline
\end{tabular}




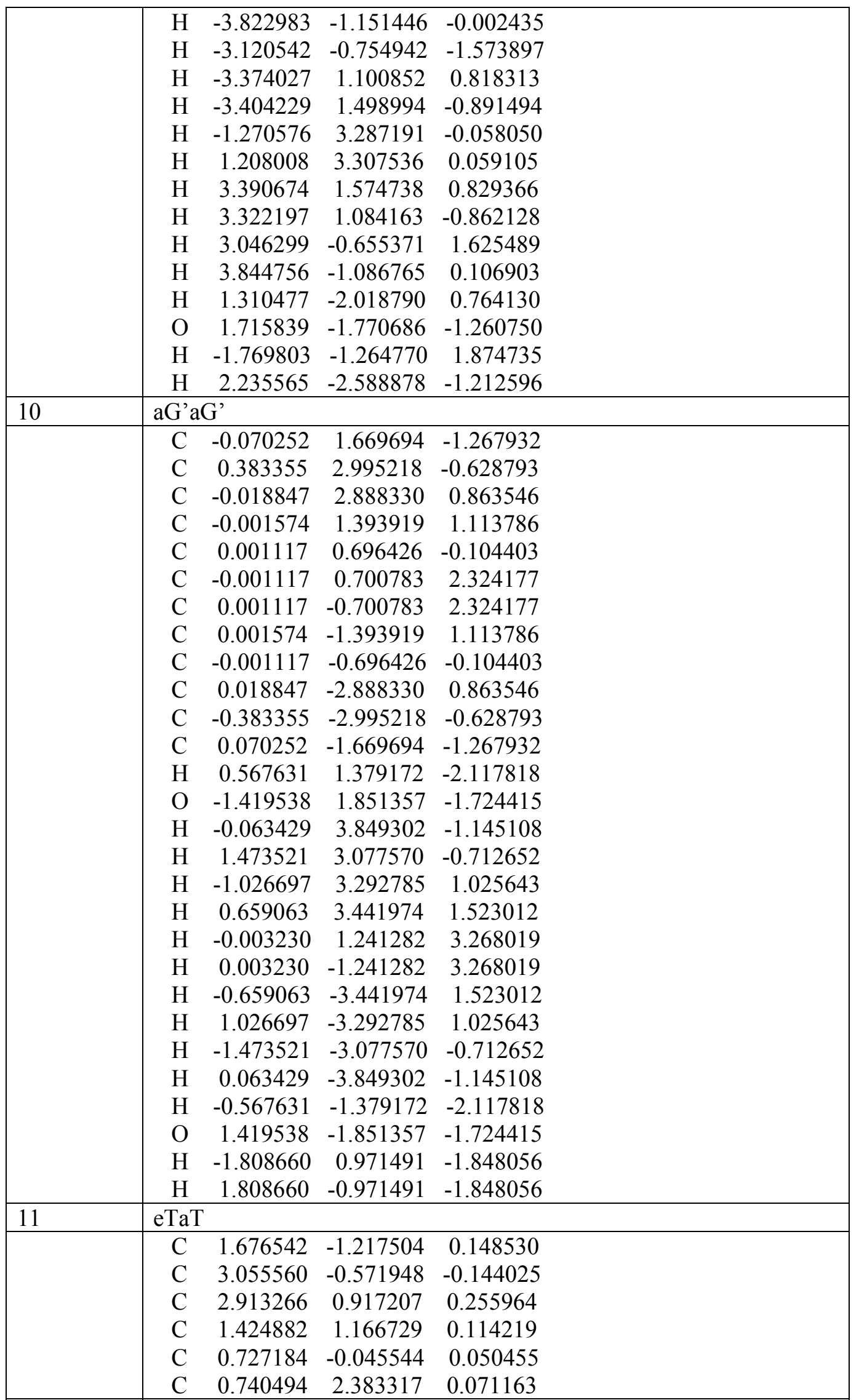




\begin{tabular}{|c|c|c|c|c|}
\hline & $\begin{array}{l}\mathrm{C} \\
\mathrm{C} \\
\mathrm{C} \\
\mathrm{C} \\
\mathrm{C} \\
\mathrm{C} \\
\mathrm{H} \\
\mathrm{O} \\
\mathrm{H} \\
\mathrm{H} \\
\mathrm{H} \\
\mathrm{H} \\
\mathrm{H} \\
\mathrm{H} \\
\mathrm{H} \\
\mathrm{H} \\
\mathrm{H} \\
\mathrm{H} \\
\mathrm{H} \\
\mathrm{O} \\
\mathrm{H} \\
\mathrm{H} \\
\end{array}$ & $\begin{array}{r}-0.654532 \\
-1.348549 \\
-0.660534 \\
-2.842771 \\
-2.934702 \\
-1.623388 \\
1.644005 \\
1.318974 \\
3.217739 \\
3.884629 \\
3.525261 \\
3.233180 \\
1.286803 \\
-1.190969 \\
-3.344770 \\
-3.311522 \\
-2.974623 \\
-3.820194 \\
-1.278341 \\
-1.728594 \\
1.827208 \\
-2.262165 \\
\end{array}$ & $\begin{array}{r}2.390391 \\
1.179630 \\
-0.039542 \\
0.941851 \\
-0.549673 \\
-1.204559 \\
-1.626716 \\
-2.243533 \\
-0.652842 \\
-1.078187 \\
1.582546 \\
1.075299 \\
3.323174 \\
3.335805 \\
1.603335 \\
1.115348 \\
-0.629469 \\
-1.054366 \\
-2.007941 \\
-1.699436 \\
-3.035240 \\
-2.509124 \\
\end{array}$ & $\begin{array}{c}-0.034391 \\
-0.093067 \\
-0.053025 \\
-0.177337 \\
-0.583295 \\
-0.081062 \\
1.171723 \\
-0.780228 \\
-1.225146 \\
0.362558 \\
-0.364316 \\
1.296122 \\
0.111246 \\
-0.073356 \\
-0.892941 \\
0.800790 \\
-1.676829 \\
-0.181339 \\
-0.741704 \\
1.266108 \\
-0.546317 \\
1.227694 \\
\end{array}$ \\
\hline 12 & \multicolumn{4}{|c|}{ aTaT } \\
\hline & $\begin{array}{l}\mathrm{C} \\
\mathrm{C} \\
\mathrm{C} \\
\mathrm{C} \\
\mathrm{C} \\
\mathrm{C} \\
\mathrm{C} \\
\mathrm{C} \\
\mathrm{C} \\
\mathrm{C} \\
\mathrm{C} \\
\mathrm{C} \\
\mathrm{H} \\
\mathrm{O} \\
\mathrm{H} \\
\mathrm{H} \\
\mathrm{H} \\
\mathrm{H} \\
\mathrm{H} \\
\mathrm{H} \\
\mathrm{H} \\
\mathrm{H} \\
\mathrm{H} \\
\mathrm{H} \\
\mathrm{H} \\
\mathrm{O}\end{array}$ & $\begin{array}{r}-0.011575 \\
0.367002 \\
-0.045082 \\
-0.008907 \\
0.006750 \\
-0.008907 \\
0.008907 \\
0.008907 \\
-0.006750 \\
0.045082 \\
-0.367002 \\
0.011575 \\
0.707265 \\
-1.341298 \\
-0.105491 \\
1.453286 \\
-1.059124 \\
0.622024 \\
-0.020725 \\
0.020725 \\
-0.622024 \\
1.059124 \\
-1.453286 \\
0.105491 \\
-0.707265 \\
1.341298\end{array}$ & $\begin{array}{r}1.642364 \\
2.998752 \\
2.886553 \\
1.392515 \\
0.694896 \\
0.699703 \\
-0.699703 \\
-1.392515 \\
-0.694896 \\
-2.886553 \\
-2.998752 \\
-1.642364 \\
1.344236 \\
1.629107 \\
3.840760 \\
3.134134 \\
3.277016 \\
3.451099 \\
1.241023 \\
-1.241023 \\
-3.451099 \\
-3.277016 \\
-3.134134 \\
-3.840760 \\
-1.344236 \\
-1.629107\end{array}$ & $\begin{array}{c}-1.246767 \\
-0.597822 \\
0.891599 \\
1.141895 \\
-0.070860 \\
2.355233 \\
2.355233 \\
1.141895 \\
-0.070860 \\
0.891599 \\
-0.597822 \\
-1.246767 \\
-2.022376 \\
-1.793712 \\
-1.115802 \\
-0.667514 \\
1.052310 \\
1.553205 \\
3.298636 \\
3.298636 \\
1.553205 \\
1.052310 \\
-0.667514 \\
-1.115802 \\
-2.022376 \\
-1.793712\end{array}$ \\
\hline
\end{tabular}




\begin{tabular}{|l|rrrr|}
\hline & $\mathrm{H}$ & -1.326629 & 2.186216 & -2.588429 \\
& $\mathrm{H}$ & 1.326629 & -2.186216 & -2.588429 \\
\hline 13 & $\mathrm{aG}$ 'aT & & \\
\hline & $\mathrm{C}$ & 1.661419 & -1.252877 & 0.070681 \\
$\mathrm{C}$ & 2.987589 & -0.597647 & 0.533455 \\
$\mathrm{C}$ & 2.885898 & 0.892040 & 0.123810 \\
$\mathrm{C}$ & 1.390601 & 1.132086 & 0.084194 \\
$\mathrm{C}$ & 0.697923 & -0.085888 & 0.061300 \\
$\mathrm{C}$ & 0.695156 & 2.341520 & 0.039933 \\
$\mathrm{C}$ & -0.703262 & 2.335652 & -0.036620 \\
$\mathrm{C}$ & -1.390449 & 1.121419 & -0.077147 \\
$\mathrm{C}$ & -0.690319 & -0.092598 & -0.032397 \\
$\mathrm{C}$ & -2.880697 & 0.862800 & -0.170793 \\
$\mathrm{C}$ & -2.946235 & -0.625973 & -0.596265 \\
$\mathrm{C}$ & -1.654331 & -1.263428 & -0.053164 \\
$\mathrm{H}$ & 1.351502 & -2.055569 & 0.759426 \\
$\mathrm{O}$ & 1.721766 & -1.772497 & -1.264640 \\
$\mathrm{H}$ & 3.860206 & -1.106844 & 0.109434 \\
$\mathrm{H}$ & 3.059126 & -0.673451 & 1.625569 \\
$\mathrm{H}$ & 3.326058 & 1.059610 & -0.868355 \\
$\mathrm{H}$ & 3.411678 & 1.553656 & 0.821692 \\
$\mathrm{H}$ & 1.232852 & 3.286809 & 0.056740 \\
$\mathrm{H}$ & -1.246543 & 3.277302 & -0.072924 \\
$\mathrm{H}$ & -3.387712 & 1.526914 & -0.880017 \\
$\mathrm{H}$ & -3.358734 & 1.010877 & 0.806793 \\
$\mathrm{H}$ & -2.944189 & -0.696962 & -1.690159 \\
$\mathrm{H}$ & -3.828865 & -1.151787 & -0.221480 \\
$\mathrm{H}$ & -1.296365 & -2.093107 & -0.677833 \\
$\mathrm{O}$ & -1.938236 & -1.739756 & 1.275421 \\
$\mathrm{H}$ & 2.321577 & -2.535820 & -1.249569 \\
$\mathrm{H}$ & -1.088579 & -1.906245 & 1.712513 \\
\hline & & & & \\
& &
\end{tabular}

\begin{tabular}{|l|l|l|}
\hline & \multicolumn{1}{|c|}{ Notation } & $\begin{array}{l}\text { Total Energy } \\
\text { (Hartree) }\end{array}$ \\
\hline 1 & eGeG' & -616.149110253 \\
\hline 2 & eG'eT & -616.140943865 \\
\hline 3 & aGeG & -616.141562335 \\
\hline 4 & aGaG & -616.142675667 \\
\hline 5 & aTeG & -616.142875670 \\
\hline 6 & eGeG & -616.142605823 \\
\hline 7 & eTaG & -616.142935597 \\
\hline 8 & aGaG' & -616.143063080 \\
\hline 9 & aTaG & -616.141473941 \\
\hline 10 & aG'aG' & -616.141001060 \\
\hline 11 & eTaT & -616.150860980 \\
\hline 12 & aTaT & -616.143686764 \\
\hline 13 & aG'aT & -616.143822033 \\
\hline
\end{tabular}




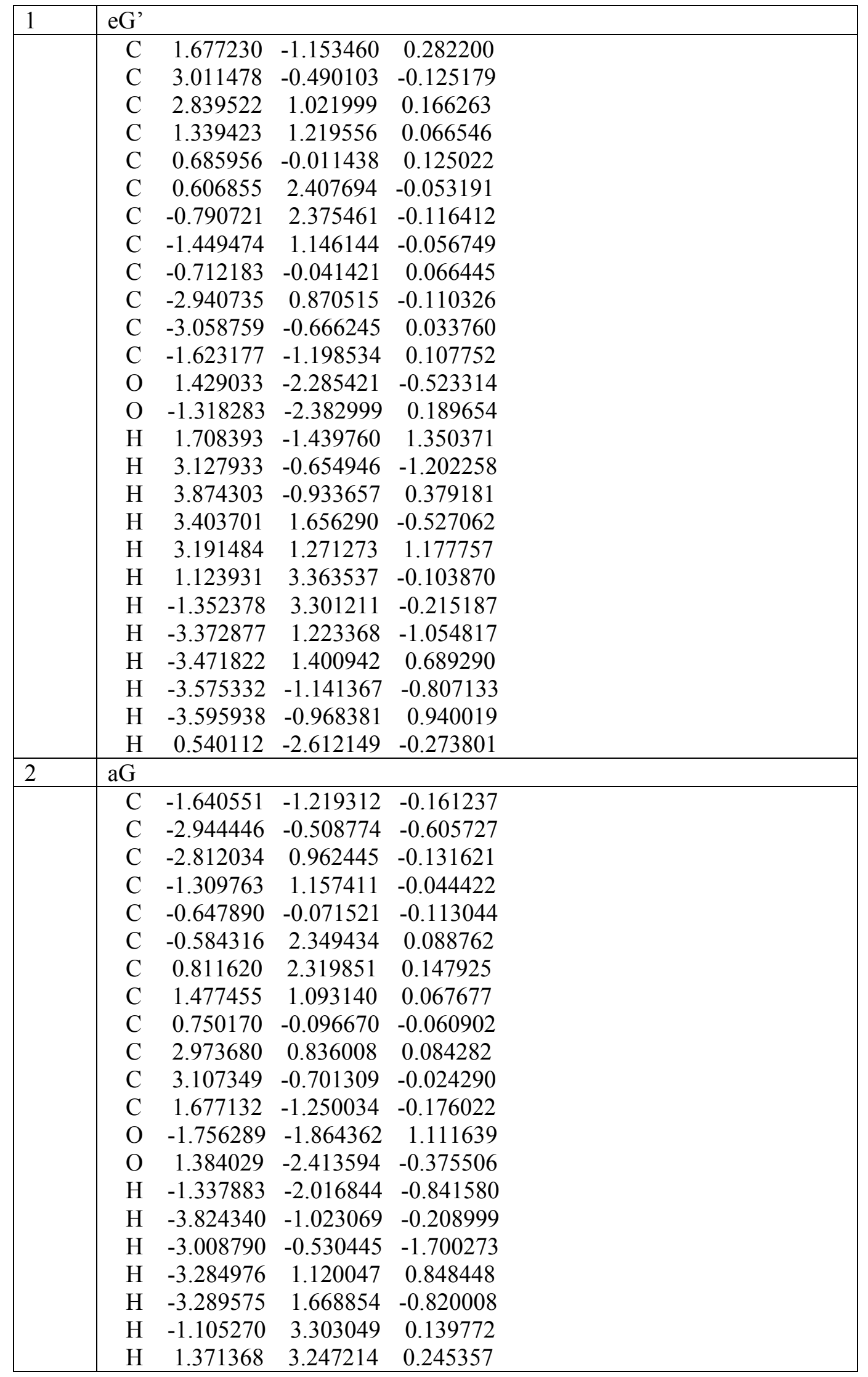




\begin{tabular}{|l|rrrr|}
\hline & $\mathrm{H}$ & 3.432884 & 1.227914 & 1.000021 \\
$\mathrm{H}$ & 3.467846 & 1.347222 & -0.751305 \\
$\mathrm{H}$ & 3.561158 & -1.148960 & 0.867372 \\
$\mathrm{H}$ & 3.711586 & -1.024702 & -0.878172 \\
$\mathrm{H}$ & -1.866366 & -1.170646 & 1.782025 \\
\hline & $\mathrm{eG}$ & & & \\
& & & & \\
$\mathrm{C}$ & -1.676566 & -1.184919 & -0.213902 \\
$\mathrm{C}$ & -3.046127 & -0.480153 & 0.016227 \\
$\mathrm{C}$ & -2.826832 & 1.026095 & -0.259085 \\
$\mathrm{C}$ & -1.331468 & 1.197258 & -0.090361 \\
$\mathrm{C}$ & -0.680090 & -0.038422 & -0.117072 \\
$\mathrm{C}$ & -0.606670 & 2.388651 & 0.042914 \\
$\mathrm{C}$ & 0.785093 & 2.354182 & 0.147394 \\
$\mathrm{C}$ & 1.445683 & 1.124179 & 0.089665 \\
$\mathrm{C}$ & 0.721184 & -0.067305 & -0.056173 \\
$\mathrm{C}$ & 2.941985 & 0.874698 & 0.140421 \\
$\mathrm{C}$ & 3.083405 & -0.660712 & 0.046677 \\
$\mathrm{C}$ & 1.664557 & -1.211994 & -0.186757 \\
$\mathrm{O}$ & -1.452615 & -2.260556 & 0.684823 \\
$\mathrm{O}$ & 1.398576 & -2.364107 & -0.464570 \\
$\mathrm{H}$ & -1.626276 & -1.649019 & -1.205530 \\
$\mathrm{H}$ & -3.318620 & -0.629992 & 1.068640 \\
$\mathrm{H}$ & -3.845778 & -0.919530 & -0.586691 \\
$\mathrm{H}$ & -3.411562 & 1.672155 & 0.406305 \\
$\mathrm{H}$ & -3.122097 & 1.286180 & -1.285296 \\
$\mathrm{H}$ & -1.127934 & 3.343097 & 0.070275 \\
$\mathrm{H}$ & 1.347043 & 3.278253 & 0.261949 \\
$\mathrm{H}$ & 3.382157 & 1.279606 & 1.059845 \\
$\mathrm{H}$ & 3.448397 & 1.379538 & -0.691891 \\
$\mathrm{H}$ & 3.477068 & -1.100756 & 0.970742 \\
$\mathrm{H}$ & 3.741218 & -0.991683 & -0.762916 \\
$\mathrm{H}$ & -1.356234 & -1.879901 & 1.572861 \\
\hline & & & & \\
& & \\
& & \\
& & \\
& & \\
& & \\
& &
\end{tabular}

\begin{tabular}{|crrr|}
\hline $\mathrm{eG} \ldots \mathrm{CHCl}_{3}$ & & \\
\hline $\mathrm{C}$ & -0.613530 & 1.371512 & 1.635752 \\
$\mathrm{C}$ & -0.155693 & 2.744827 & 1.099560 \\
$\mathrm{C}$ & -1.278794 & 3.213554 & 0.138441 \\
$\mathrm{C}$ & -1.907976 & 1.910412 & -0.314550 \\
$\mathrm{C}$ & -1.523216 & 0.870615 & 0.530104 \\
$\mathrm{C}$ & -2.771409 & 1.664612 & -1.390833 \\
$\mathrm{C}$ & -3.255835 & 0.374065 & -1.630364 \\
$\mathrm{C}$ & -2.872523 & -0.669997 & -0.785754 \\
$\mathrm{C}$ & -2.011393 & -0.418912 & 0.292283 \\
$\mathrm{C}$ & -3.260181 & -2.135411 & -0.853760 \\
$\mathrm{C}$ & -2.552285 & -2.783617 & 0.361314 \\
$\mathrm{C}$ & -1.748061 & -1.665572 & 1.029911 \\
$\mathrm{O}$ & 0.502632 & 0.537794 & 1.917848 \\
$\mathrm{O}$ & -1.021213 & -1.809025 & 2.008131 \\
\hline
\end{tabular}




\begin{tabular}{|rrrr|}
\hline $\mathrm{H}$ & -1.208338 & 1.501112 & 2.556469 \\
$\mathrm{H}$ & 0.771276 & 2.586372 & 0.536973 \\
$\mathrm{H}$ & 0.055802 & 3.459315 & 1.899541 \\
$\mathrm{H}$ & -0.898885 & 3.819095 & -0.691924 \\
$\mathrm{H}$ & -2.018128 & 3.831667 & 0.667538 \\
$\mathrm{H}$ & -3.063800 & 2.477982 & -2.051183 \\
$\mathrm{H}$ & -3.919649 & 0.190693 & -2.471598 \\
$\mathrm{H}$ & -2.933351 & -2.584571 & -1.799429 \\
$\mathrm{H}$ & -4.348749 & -2.260936 & -0.811240 \\
$\mathrm{H}$ & -1.873369 & -3.596848 & 0.083631 \\
$\mathrm{H}$ & -3.256092 & -3.200161 & 1.090971 \\
$\mathrm{H}$ & 0.131178 & -0.333416 & 2.183061 \\
$\mathrm{H}$ & 1.898265 & 0.033366 & 0.508644 \\
$\mathrm{C}$ & 2.629746 & -0.263980 & -0.245254 \\
$\mathrm{Cl}$ & 3.036715 & 1.189253 & -1.216191 \\
$\mathrm{Cl}$ & 4.074692 & -0.902016 & 0.590918 \\
$\mathrm{Cl}$ & 1.873262 & -1.524089 & -1.275339 \\
\hline
\end{tabular}

\begin{tabular}{|l|l|l|}
\hline & Notation & $\begin{array}{l}\text { Total Energy } \\
\text { (Hartree) }\end{array}$ \\
\hline 1 & eG' & -614.952762088 \\
\hline 2 & aG & -614.950634805 \\
\hline 3 & eG & -614.963678955 \\
\hline & eG'... $\mathrm{CHCl}_{3}$ & -2034.25337174 \\
\hline
\end{tabular}




\begin{tabular}{|rrrr|}
\hline C & -1.663323 & -1.374757 & -0.132370 \\
C & -0.690501 & -0.247115 & -0.067467 \\
C & -1.386433 & 0.976650 & -0.136910 \\
C & -2.877343 & 0.742050 & -0.330290 \\
C & -3.006475 & -0.778285 & -0.280002 \\
C & 0.690501 & -0.247115 & 0.067468 \\
C & 1.386433 & 0.976650 & 0.136910 \\
C & 0.699295 & 2.183802 & 0.064669 \\
C & -0.699295 & 2.183802 & -0.064669 \\
C & 1.663323 & -1.374758 & 0.132368 \\
C & 3.006474 & -0.778286 & 0.280003 \\
C & 2.877343 & 0.742050 & 0.330290 \\
O & 2.609005 & -1.355198 & -0.969568 \\
O & -2.609004 & -1.355199 & 0.969568 \\
H & 3.825585 & -1.283799 & 0.788213 \\
H & 3.485305 & 1.217452 & -0.449305 \\
$\mathrm{H}$ & 3.225825 & 1.128644 & 1.297473 \\
$\mathrm{H}$ & 1.236237 & 3.127971 & 0.117221 \\
$\mathrm{H}$ & -1.236237 & 3.127971 & -0.117222 \\
$\mathrm{H}$ & -3.225827 & 1.128646 & -1.297471 \\
$\mathrm{H}$ & -3.485304 & 1.217452 & 0.449306 \\
$\mathrm{H}$ & -3.825585 & -1.283801 & -0.788212 \\
$\mathrm{H}$ & -1.393982 & -2.362749 & -0.497334 \\
$\mathrm{H}$ & 1.393981 & -2.362750 & 0.497330 \\
\hline-613.663105487 & & \\
\hline
\end{tabular}


$(1 S, 8 S)-1,2,3,6,7,8$-Hexahydro-as-indacene-1,8-diol $\quad 1 \quad$ and $\quad(8 S)$-8-hydroxy-3,6,7,8tetrahydro-2H-as-indacen-1-one 2:

A mixture of $\left[\mathrm{RuCl}_{2}\left(\eta^{6} \text {-cymene }\right)\right]_{2},(226 \mathrm{mg}, 0.369 \mathrm{mmol}),(S, S)$-Ts-DPEN (282 mg, 0.770 mmol) and $\mathrm{NEt}_{3}(145 \mu \mathrm{l}, 1.04 \mathrm{mmol})$ was dissolved in degassed DMF (70 mL) and stirred for $1 \mathrm{~h}$ at $80^{\circ} \mathrm{C}$ in a closed vessel under argon. At room temperature, this solution was added to 4 (4.71 g, $0.0253 \mathrm{~mol})$ in a flask equipped with an argon balloon. After addition of $\mathrm{HCOOH}: \mathrm{NEt}_{3}(5: 2$ azeotrope; $35 \mathrm{~mL})$ the mixture was stirred for $96 \mathrm{~h}$. The solution was poured into $\mathrm{H}_{2} \mathrm{O}(500 \mathrm{~mL})$ and extracted with toluene $(8 \times 500 \mathrm{~mL})$. After drying with $\mathrm{Na}_{2} \mathrm{SO}_{4}$, filtration and evaporation of the volatiles, the crude material (5.72 $\mathrm{g}$ ) was purified by chromatography on silica gel with gradient elution (iPrOH/toluene, 1-5\%) resulting in three fractions: $0.43 \mathrm{~g}$ of an apolar fraction containing (8S)-2, $0.134 \mathrm{~g}$ meso-1 (0.704 mmol, $2 \%)$ and $4.2 \mathrm{~g}$ of a more polar fraction containing $(1 S, 8 S)-\mathbf{1}(>99 \%$ ee). The polar fraction was further purified by crystallisation from nhexane/benzene 50/50 resulting in $3.905 \mathrm{~g}(1 \mathrm{~S}, 8 \mathrm{~S})$-1 (20.53 mmol, $81 \%,>99 \%$ ee). The apolar fraction was further purified by chromatography on silica gel (pentane/acetone, 90/10) resulting in $247 \mathrm{mg}(8 S)-2(1.312 \mathrm{mmol}, 5 \%, 98 \%$ ee)

Data for $(1 S, 8 S)$-1 from ref. 15: For analytical purposes the product was crystallized twice from $\mathrm{CH}_{2} \mathrm{Cl}_{2 .} \mathrm{R}_{\mathrm{f}}: 0.20$ (tolueen/iPrOH 95:5), $\mathrm{mp}=155.5-156.5^{\circ} \mathrm{C} ;[\alpha]_{\mathrm{D}}^{20}=+70.5(\mathrm{c} 1.09$, $\left.\mathrm{CHCl}_{3}\right) ;{ }^{1} \mathrm{H} \mathrm{NMR}\left(500 \mathrm{MHz}, \mathrm{CDCl}_{3}\right): \delta(\mathrm{ppm})=1.91(2 \mathrm{H}$, dddd [app. dq], J= 8.4, 9.3, 9.3, 12.5), $2.56(2 \mathrm{H}$, dddd, $\mathrm{J}=2.0,7.1,7.5,12.5), 2.77(2 \mathrm{H}, \mathrm{ddd}, \mathrm{J}=7.5,9.3,15.0), 2.94(2 \mathrm{H}$, $\mathrm{ddd}, \mathrm{J}=2.0,9.3,15.0), 5.47(2 \mathrm{H}, \mathrm{dd}[$ app. $\mathrm{t}], \mathrm{J}=7.1,8.4), 7.10(2 \mathrm{H}, \mathrm{s}) ;{ }^{13} \mathrm{C} \mathrm{NMR}(125 \mathrm{MHz}$ $\left.\mathrm{CDCl}_{3}\right): \delta(\mathrm{ppm})=29.7\left(\mathrm{CH}_{2}\right), 35.9\left(\mathrm{CH}_{2}\right), 75.6(\mathrm{CH}), 124.5(\mathrm{CH}), 140.6(\mathrm{C}), 141.1(\mathrm{C}) \mathrm{ppm}$ MS (m/z, \%): 115 (40), 128 (67), 129 (100), 144 (74), 153 (48), 154 (34), 171 (28), 172 (64),

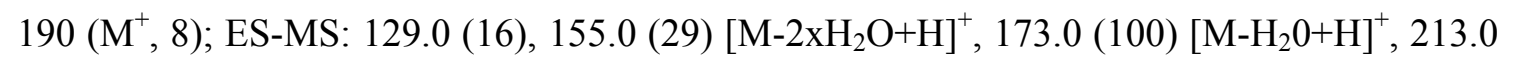


(6) $[\mathrm{M}+\mathrm{Na}]^{+}, \mathrm{IR}\left(\mathrm{CDCl}_{3}\right) 2360,2340,2254,1794,1643,1561,1469,1383,1329,1230,1166$, $1095,1075,918,909,902,736,651,625,543 \mathrm{~cm}^{-1}$. Elemental analysis. Calculated for $\mathrm{C}_{12} \mathrm{H}_{14} \mathrm{O}_{2}:$ C, 75.76; H, 7.42 Found: C, 75.68; H, 7.51

HPLC: Chiralcel OD-H column, solvent: $n$-hexane $/ \mathrm{EtOH}(97: 3)$, flow rate $=1 \mathrm{~mL} / \mathrm{min}, \mathrm{T}=$ $35^{\circ} \mathrm{C}$, retention times: $(1 S, 8 S)-\mathbf{1}=14.2 \mathrm{~min},(1 R, 8 R)-\mathbf{1}=16.1 \mathrm{~min}$.

Data for (8S)-2 from ref. 15: For analytical purposes the product was crystallized twice from diisopropyl ether. $\mathrm{R}_{\mathrm{f}}$ : $0.16\left(\mathrm{CH}_{2} \mathrm{Cl}_{2} / \mathrm{EtOAc} 98: 2\right), \mathrm{mp}=108-110^{\circ} \mathrm{C} ;[\alpha]_{D}^{25}=+98.2$ (c 1.04, $\mathrm{CHCl}_{3},>99 \%$ ee); ${ }^{1} \mathrm{H} \mathrm{NMR}\left(500 \mathrm{MHz} ; \mathrm{CDCl}_{3}\right): \delta(\mathrm{ppm})=2.03(1 \mathrm{H}$, dddd [app. dq], $\mathrm{J}=8.2,9.5,9.5,13.0 \mathrm{~Hz}), 2.61(\mathrm{dddd}, 1 \mathrm{H}, \mathrm{J}=1.8,7.8,8.0,13.0), 2.69-2.80(2 \mathrm{H}, \mathrm{m}), 2.87(1 \mathrm{H}$, ddd, $\mathrm{J}=8.0,9.5,15.9), 3.04(1 \mathrm{H}, \mathrm{ddd}, \mathrm{J}=1.8,9.5,15.9 \mathrm{~Hz}), 3.14-3.23(2 \mathrm{H}, \mathrm{m}), 5.58(1 \mathrm{H}, \mathrm{dd}$, $\mathrm{J}=7.8,8.2 \mathrm{~Hz}), 7.34(1 \mathrm{H}, \mathrm{d}, \mathrm{J}=7.8 \mathrm{~Hz}), 7.44(1 \mathrm{H}, \mathrm{d}, \mathrm{J}=7.8 \mathrm{~Hz}) ;{ }^{13} \mathrm{C}$ NMR $(125 \mathrm{MHz}$, $\left.\mathrm{CDCl}_{3}\right): \delta(\mathrm{ppm})=26.6\left(\mathrm{CH}_{2}\right), 30.3\left(\mathrm{CH}_{2}\right), 33.9\left(\mathrm{CH}_{2}\right), 36.5\left(\mathrm{CH}_{2}\right), 75.2(\mathrm{CH}), 126.0(\mathrm{CH})$, $131.7(\mathrm{CH}), 133.5$ (C), 142.2 (C), 144.8 (C), 154.8 (C), 210.2 (C); MS (m/z, \%):188 (M+, 52), 187 (25), 160 (100), 159 (59), 132 (28), 128 (27), 115 (31), 103 (9), 91 (14), 77 (17), 63 (14), 51 (18); ES-MS: 129.0 (10), 171.0 (100) [M- $\left.\mathrm{H}_{2} 0+\mathrm{H}\right]^{+}, 211.0$ (4) $[\mathrm{M}+\mathrm{Na}]^{+}$; IR $\left(\mathrm{CDCl}_{3}\right)$ 2937, $2248,1687,1614,1587,1473,1452,1438,1403,1333,1287,1282,1253,1236,1094,1069$, 920, 909, 904, 899, 839, 752, 740, 731, 647, $590 \mathrm{~cm}^{-1}$. Elemental analysis. Calculated for $\mathrm{C}_{12} \mathrm{H}_{12} \mathrm{O}_{2}:$ C, 76.57; H, 6.43 Found: C, 76.46; H, 6.57.

HPLC: Chiralcel OD-H column, solvent: -hexane/EtOH (98:2), flow rate $=1 \mathrm{~mL} / \mathrm{min}, \mathrm{T}=$ $35^{\circ} \mathrm{C}$, retention times $=(8 S)-2=11.5 \mathrm{~min},(8 R)-2=12.8 \mathrm{~min}$.

Data for meso-diol see ref. 15 

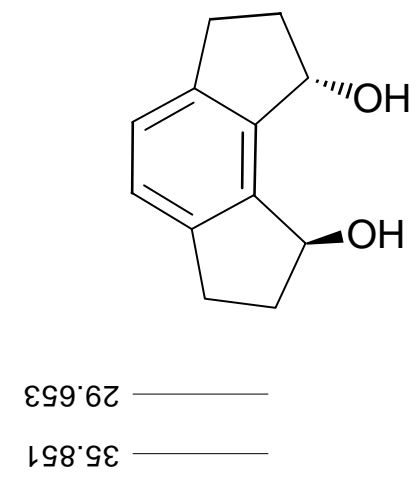

OโG'จ乙โ

6ย9.0๑

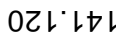

629.9L
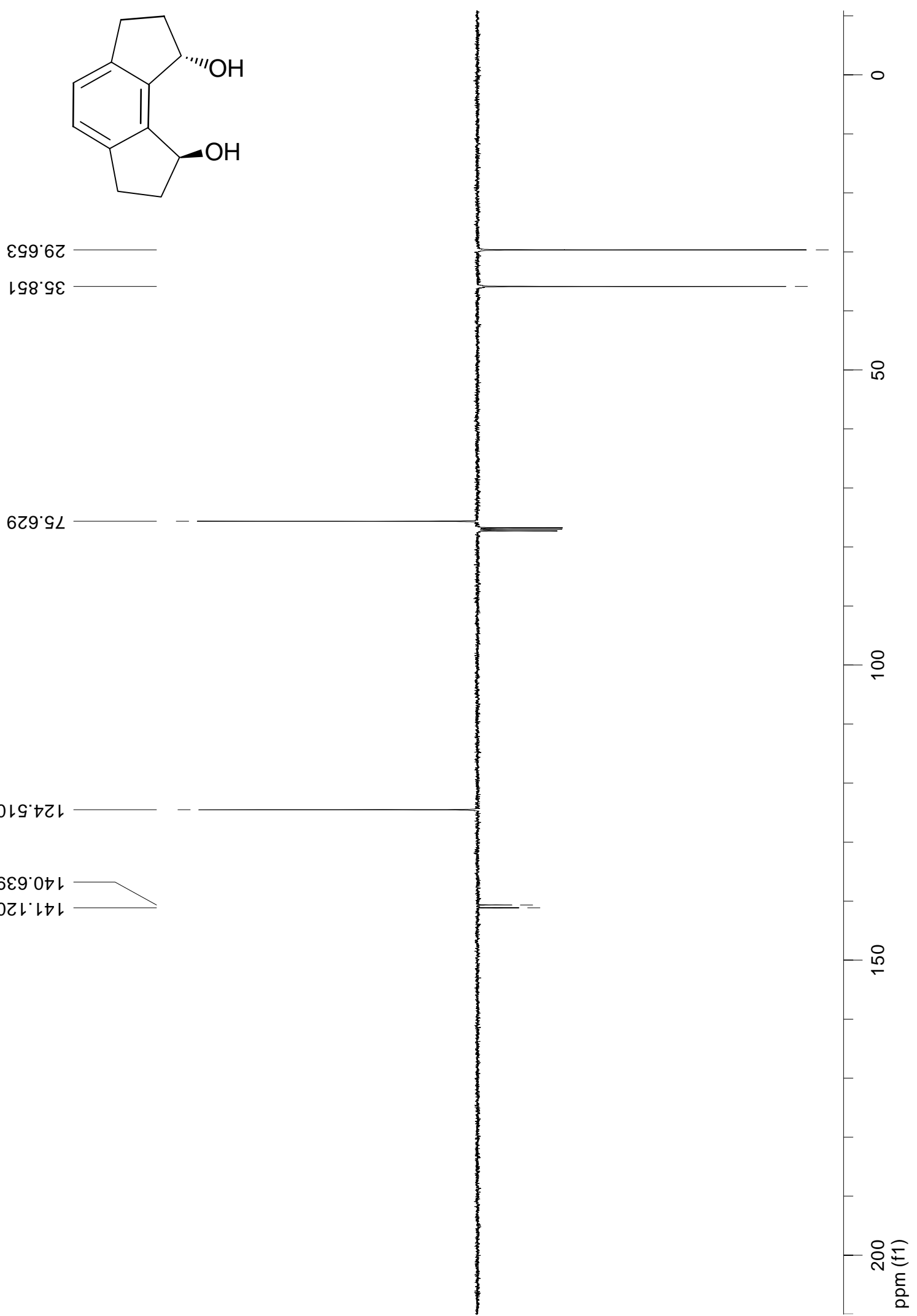


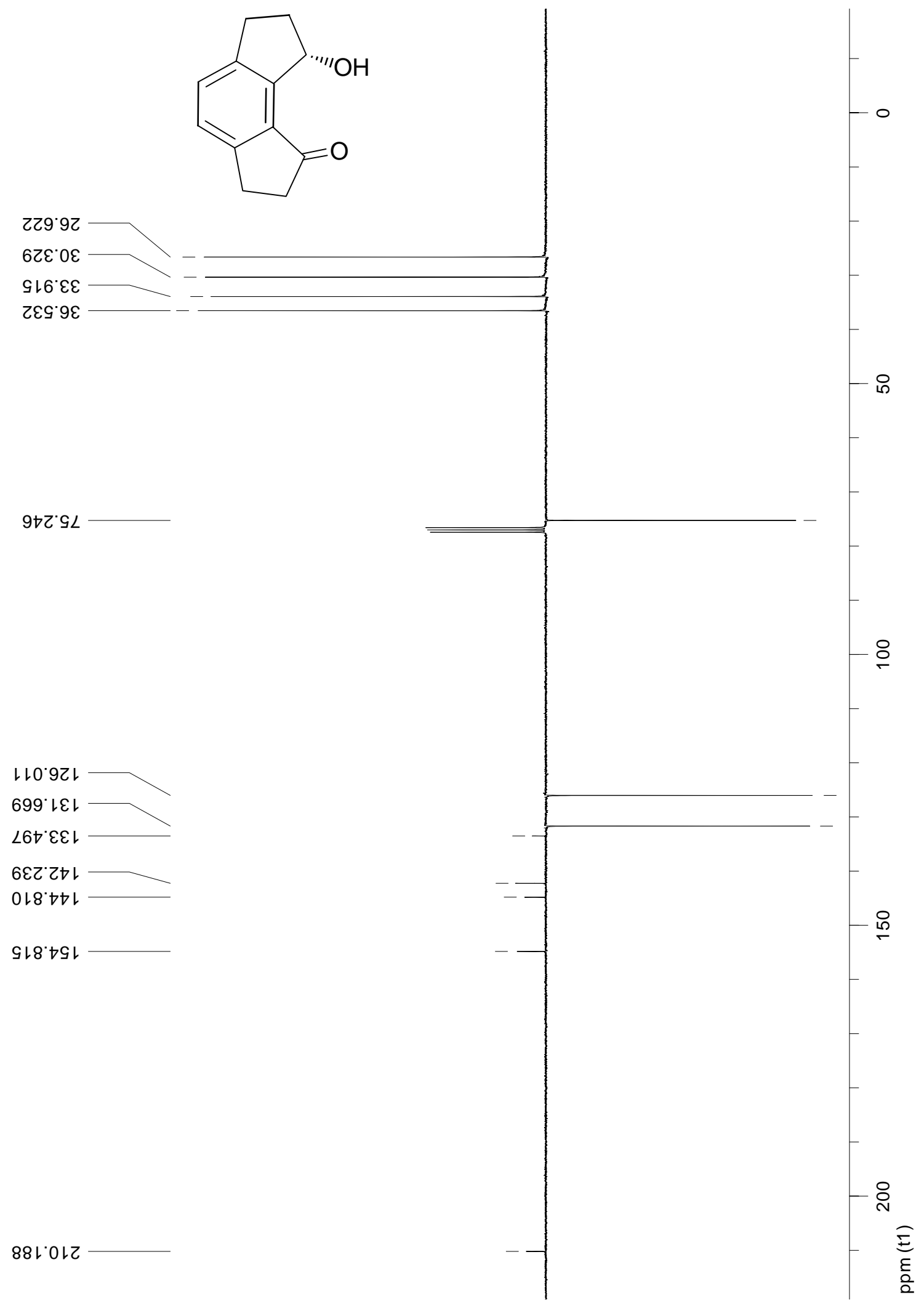




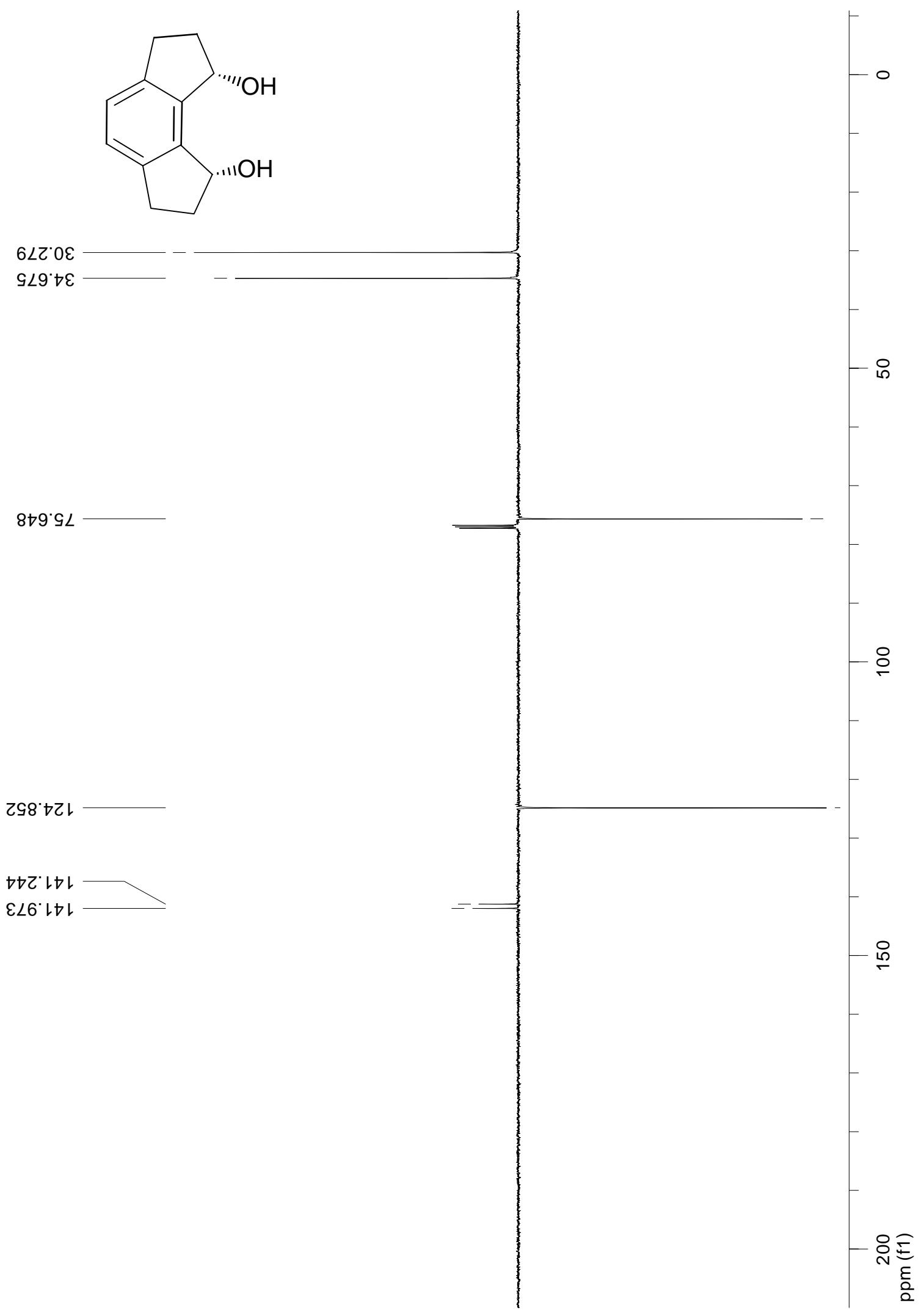

